\title{
Momente der Klassenzahlen binärer quadratischer Formen mit ganzalgebraischen Koeffizienten
}

von

\author{
MAnFRed Peter (Freiburg)
}

1. Einleitung und Formulierung des Ergebnisses. Für die Anzahl $h(d)$ der Äquivalenzklassen primitiver binärer quadratischer Formen zur Diskriminante $d$ beweist Siegel [22]

$$
\sum_{1<d \leq x} h(d) \log \varepsilon_{d}=\frac{\pi^{2}}{18 \zeta(3)} x^{3 / 2}+O(x \log x),
$$

wobei $\varepsilon_{d}>1$ die Fundamentallösung der Pellschen Gleichung $u^{2}-d v^{2}=4$ ist. Für $d \equiv 0$ (4) wurde eine analoge Formel von Gauß vermutet ("Disquisitiones Arithmeticae"); siehe auch I. M. Vinogradov [27], Mertens [13], I. M. Vinogradov [26], Shintani [20], Hooley [8]. Die Formel

$$
\sum_{\varepsilon_{d} \leq x} h(d) \log \varepsilon_{d}=\frac{1}{2} x^{2}+O\left(x^{3 / 2} \log ^{3} x\right)
$$

wurde von Sarnak [16] mit Hilfe der Selbergschen Zetafunktion für diskontinuierliche Gruppen bewiesen; siehe auch Iwaniec [9] und Sarnak [18]. In [17] beweist Sarnak die Formel (1) für quadratische Formen, deren Koeffizienten ganze Zahlen aus einem imaginärquadratischen Zahlkörper mit Klassenzahl 1 sind. Wolke [28] beweist die Formel

$$
\sum_{n \leq x} h(-n)^{\alpha}=\Theta(\alpha) x^{(\alpha+2) / 2}+O\left(x^{(\alpha+2) / 2-1 / 4+\varepsilon}\right) \quad(\alpha, \varepsilon>0)
$$

(vgl. Barban [1]).

Ziel der vorliegenden Arbeit ist folgende Verallgemeinerung von (1): $k$ sei totalreeller Zahlkörper mit ungerader Klassenzahl. Sei $\mathcal{O}_{k}$ der Ring der ganzen Zahlen in $k, E\left(\mathcal{O}_{k}\right)$ seine Einheitengruppe, $\sigma_{i}: k \rightarrow \mathbb{R}, 1 \leq i \leq n:=$ $[k: \mathbb{Q}]$, seine $\mathbb{Q}$-Einbettungen und o.E. $\sigma_{1}=\mathrm{id}_{k}, k \subset \mathbb{R}$.

Auf der Menge

$\widetilde{\mathcal{D}}:=\left\{D \in \mathcal{O}_{k} \mid x^{2} \equiv D(4)\right.$ in $\mathcal{O}_{k}$ lösbar, $D$ kein Quadrat in $\mathcal{O}_{k}$,

$$
\left.\sigma_{1}(D)>0, \sigma_{2}(D), \ldots, \sigma_{n}(D)<0\right\}
$$


werde die Äquivalenzrelation $\sim$ durch

$$
D_{1} \sim D_{2}: \Leftrightarrow D_{1}=e^{2} D_{2} \text { für ein } e \in E\left(\mathcal{O}_{k}\right)
$$

definiert; $\mathcal{D} \subset \widetilde{\mathcal{D}}$ sei ein vollständiges Repräsentantensystem von $\sim$.

Für $D \in \widetilde{\mathcal{D}}$ definiere man die Gruppe

$$
U_{D}:=\left\{\frac{1}{2}(x+y \sqrt{D}) \mid x, y \in \mathcal{O}_{k}, x^{2}-D y^{2}=4\right\} .
$$

In (33) wird gezeigt, daß es eine direkte Zerlegung $U_{D}=\{ \pm 1\} \odot\left\langle\varepsilon_{D}\right\rangle$ mit eindeutig bestimmtem $\varepsilon_{D}>1$ gibt. Ist $D \in \mathcal{O}_{k}, x^{2} \equiv D$ (4) lösbar, so heißt (3) $a x^{2}+b x y+c y^{2} \quad$ mit $a, b, c \in \mathcal{O}_{k}, a \mathcal{O}_{k}+b \mathcal{O}_{k}+c \mathcal{O}_{k}=\mathcal{O}_{k}, D=b^{2}-4 a c$ primitive quadratische Form mit Diskriminante $D$.

Äquivalenz von quadratischen Formen (3) ist wie üblich bezüglich linearer Variablentransformationen mit Koeffizientenmatrizen $\left(\begin{array}{l}p q \\ r\end{array}\right), p, q, r, s \in$ $\mathcal{O}_{k}, p s-q r=1$, definiert; die Anzahl der Äquivalenzklassen werde mit $h(D)$ bezeichnet.

Sei $m \in \mathbb{N}, \varepsilon>0$.

SATZ 1.1. Mit einer nur von $n$ abhängigen Konstante $\varrho<1$ (siehe (74)) und einem von $k$ und $m$ abhängigen $\lambda_{m}>0$ gilt für $x \rightarrow \infty$

$$
\Theta_{m}(x):=\sum_{D \in \mathcal{D}: \varepsilon_{D} \leq x}\left(h(D) \log \varepsilon_{D}\right)^{m}=\lambda_{m} x^{m+1}+O\left(x^{m+\varrho+\varepsilon}\right) .
$$

Die zum Beweis verwendete Methode geht auf Sarnak [18] zurück.

Für $x \geq 1$ sei $N(x):=\left|\left\{D \in \mathcal{D} \mid \varepsilon_{D} \leq x\right\}\right|$. Analog zu Satz 1.1 beweist man

$$
\sum_{D \in \mathcal{D}: \varepsilon_{D} \leq x}\left(\frac{h(D) \log D}{D^{1 / 2}}\right)^{m}=\mu_{m} x+O\left(\frac{x}{\log x}\right) .
$$

Wie bei Barban [1] kann daraus gefolgert werden:

SATZ 1.2. Es existiert eine Verteilungsfunktion $F: \mathbb{R} \rightarrow[0,1]$, sodaß in jedem Stetigkeitspunkt $z$ von $F$ für $x \rightarrow \infty$ gilt

$$
\frac{1}{N(x)}\left|\left\{D \in \mathcal{D} \mid \varepsilon_{D} \leq x, \frac{h(D) \log D}{D^{1 / 2}} \leq z\right\}\right| \rightarrow F(z) .
$$

Diese Arbeit ist eine Zusammenfassung meiner Doktorarbeit. Ich danke Herrn Prof. Wolke für die Betreuung meiner Arbeit und Herrn Prof. Hinz für seine Kommentare zu einer vorläufigen Version.

2. Die Dirichletsche Klassenzahlformel über Zahlkörpern. In diesem Abschnitt wird nur vorausgesetzt, daß $k$ Zahlkörper mit ungerader Klassenzahl $h_{k}$ ist (das geht in Lemma 2.11 und (41) ein). Seien $\sigma_{1}, \ldots, \sigma_{r}$ die reellen und $\sigma_{r+1}, \bar{\sigma}_{r+1}, \ldots, \sigma_{r+s}, \bar{\sigma}_{r+s}$ die komplexen $\mathbb{Q}$-Einbettungen von $k$ in $\mathbb{C}(n=r+2 s)$. 
Für eine Diskriminante $D \in \mathcal{O}_{k}$ (d.h. $X^{2} \equiv D(4)$ soll in $\mathcal{O}_{k}$ lösbar sein), $D$ kein Quadrat, definiere man $K:=k(\sqrt{D})$; für ein Primideal $\mathfrak{p} \neq 0$ in $\mathcal{O}_{k}$ mit $\mathfrak{p}^{a} \| 2$ sei

$$
\begin{aligned}
& \chi_{D}(\mathfrak{p}):=\left(\frac{D}{\mathfrak{p}}\right):= \begin{cases}1, & \mathfrak{p} \nmid D, x^{2} \equiv D\left(\mathfrak{p}^{2 a+1}\right) \text { lösbar, } \\
-1, & \mathfrak{p} \nmid D, x^{2} \equiv D\left(\mathfrak{p}^{2 a+1}\right) \text { unlösbar, } \\
0, & \mathfrak{p} \mid D,\end{cases} \\
& \chi_{D}^{*}(\mathfrak{p}):= \begin{cases}1, & \mathfrak{p} \text { spaltet in } K \text { auf }\left(\mathfrak{p} \mathcal{O}_{k}=\mathfrak{P}_{1} \mathfrak{P}_{2}, \mathfrak{P}_{1} \neq \mathfrak{P}_{2}\right), \\
-1, & \mathfrak{p} \text { ist in } K \text { träge }\left(\mathfrak{p} \mathcal{O}_{k}=\mathfrak{P}\right), \\
0, & \mathfrak{p} \text { ist in } K \text { verzweigt }\left(\mathfrak{p} \mathcal{O}_{k}=\mathfrak{P}^{2}\right),\end{cases}
\end{aligned}
$$

$\chi_{D}$ bzw. $\chi_{D}^{*}$ wird multiplikativ auf die zu $D$ bzw. zur Diskriminante $d_{K / k}$ von $K / k$ teilerfremden gebrochenen Ideale von $k$ fortgesetzt; auf den übrigen wird der Wert 0 definiert. Bei Hilbert [6], §5, Sätze 6-8, sind die folgenden Eigenschaften zu finden:

Lemma 2.1. (a) $\chi_{D}^{*}(\mathfrak{p})=\chi_{D}(\mathfrak{p})$ für Primideale $\mathfrak{p} \nmid D$.

(b) $\mathfrak{p}$ verzweigt in $K \Leftrightarrow \mathfrak{p} \mid d_{K / k}$ für beliebige Primideale $\mathfrak{p} \neq 0$ in $\mathcal{O}_{k}$.

Wie im Fall der Riemannschen Zetafunktion und Dirichletscher $L$-Reihen beweist man

Lemma 2.2. Sind $\zeta_{K}$ bzw. $\zeta_{k}$ die Dedekindschen Zetafunktionen von $K$ bzw. $k$ und ist

$$
L\left(s, \chi_{D}^{*}\right):=\sum_{\mathfrak{a}} \frac{\chi_{D}^{*}(\mathfrak{a})}{N_{k / \mathbb{Q}}(\mathfrak{a})^{s}} \quad(\Re s>1),
$$

wobei $\mathfrak{a}$ alle ganzen Ideale $\neq 0$ von $\mathcal{O}_{k}$ durchläuft, so gilt

$$
L\left(s, \chi_{D}^{*}\right)=\prod_{\mathfrak{p}}\left(1-\frac{\chi_{D}^{*}(\mathfrak{p})}{N(\mathfrak{p})^{s}}\right)^{-1}, \quad \zeta_{K}(s)=\zeta_{k}(s) L\left(s, \chi_{D}^{*}\right) \quad(\Re s>1) .
$$

In der Terminologie von Landau [11] folgt aus dem Artinschen Reziprozitätsgesetz im Spezialfall relativquadratischer Erweiterungen:

Lemma 2.3. $\chi_{D}$ ist Idealklassencharakter $\bmod D \mathcal{O}_{k}$ im engeren Sinn, ist nicht der Hauptcharakter und wird von $\chi_{D}^{*}$ induziert. $\chi_{D}^{*}$ ist primitiver Idealklassencharakter. $\chi_{D}$ und $\chi_{D}^{*}$ haben den Führer $d_{K / k}$.

Ziel dieses Abschnitts ist der Beweis von

Proposition 2.4. Ist die Klassenzahl von $k$ ungerade, so gilt

$$
h(D)=\left|d_{k / \mathbb{Q}} N_{k / \mathbb{Q}}(D)\right|^{1 / 2} \frac{\left|T\left(U_{D}\right)\right|}{2^{p} \pi^{n-p} R(D)} L\left(1, \chi_{D}\right),
$$

wobei $R(D)$ durch (30) definiert ist, $p:=\operatorname{rang} U_{D}$ und

$$
L\left(s, \chi_{D}\right):=\sum_{\mathfrak{a}} \frac{\chi_{D}(\mathfrak{a})}{N_{k / \mathbb{Q}}(\mathfrak{a})^{s}} \quad(\Re s>1) .
$$


Da ich keinen Beweis von Proposition 2.4 in der Literatur finden konnte (Dirichlet [3] untersucht $k=\mathbb{Q}(\sqrt{-1})$, Speiser [23] legt einen anderen Äquivalenzbegriff zugrunde, Shyr [21] behandelt algebraische Tori), wird im Folgenden ein Beweis skizziert, der eine Übertragung des Beweises von Zagier [29], S. 92ff, und S. 95, Aufgabe 5, sowie Borewicz-Šafarevič [2], S. 153ff und S. 170, Aufgaben 6-11, ist. Dabei wird der allgemeine Fall durch Lokalisierung bezüglich Primidealen auf den Spezialfall $h_{k}=1$ zurückgeführt.

Daher wird alles für einen beliebigen Dedekindring $\mathcal{O}_{k} \subset A \subset k$ durchgeführt. Sei $B:=A_{[K]}$ der ganze Abschluß von $A$ in $K$. Die Lokalisierung eines $A$-Moduls $M \subset K$ bezüglich eines Primideals $\mathfrak{p}$ von $\mathcal{O}_{k}$ wird mit $M_{\mathfrak{p}}$ bezeichnet. Es gilt

$$
M=\bigcap_{\mathfrak{p}} M_{\mathfrak{p}}
$$

wobei $\mathfrak{p}$ alle maximalen Ideale von $A$ durchläuft. $\alpha \rightarrow \alpha^{\prime}$ bezeichne die $k$-Konjugation auf $K$.

Nach O'Meara [15], Theorem (81:3), existiert eine $k$-Basis $1, \omega$ von $K$ und ein ganzes Ideal $\mathfrak{b}$ von $A$ mit

$$
B=A \oplus \mathfrak{b} \omega .
$$

Durch Lokalisieren und verwenden der Formel für die Diskriminante freier $A_{\mathfrak{p}}$-Moduln erhält man

$$
d_{K / k}=\mathfrak{b}^{2}\left(\omega-\omega^{\prime}\right)^{2} .
$$

Sei $u \in \mathcal{O}_{k}$ mit $u^{2} \equiv D(4)$. Dann ist $\widetilde{M}:=A \oplus A \frac{u+\sqrt{D}}{2}$ freier $A$-Modul in $B$ und mit dem ganzen Ideal $\mathfrak{c}:=N_{K / k}(\widetilde{M})$ gilt

$$
D A=\left|\begin{array}{cc}
1 & \frac{u+\sqrt{D}}{2} \\
1 & \frac{u-\sqrt{D}}{2}
\end{array}\right|^{2} A=d_{K / k}(\widetilde{M})=N_{K / k}(\widetilde{M})^{2} d_{K / k}=\mathfrak{c}^{2} d_{K / k} .
$$

Sei $M \subset K A$-Netz (endlich erzeugter $A$-Modul vom Rang 2) und $\mathcal{O}:=$ $\{\alpha \in K \mid \alpha M \subset M\}$ sein Multiplikatorenring; für ihn gilt

$$
\mathcal{O}(M) \subset B, \quad \mathcal{O}(M)_{\mathfrak{p}}=\mathcal{O}\left(M_{\mathfrak{p}}\right) \quad \text { für jedes Primideal } \mathfrak{p} \text { von } A,
$$

und ferner ist $\mathcal{O}(M) A$-Ordnung (d.h. $A$-Netz und Ring mit Eins). Der von der Menge

$$
\begin{array}{r}
\left\{\operatorname{det}\left(a_{i j}\right) \in k^{*} \mid \alpha_{i}, \beta_{i} \in K, \beta_{1} A \oplus \beta_{2} A \subset M, \alpha_{1} A \oplus \alpha_{2} A \supset \mathcal{O}(M),\right. \\
\left.\beta_{i}=a_{i 1} \alpha_{1}+a_{i 2} \alpha_{2}, a_{i j} \in k, i=1,2\right\}
\end{array}
$$

erzeugte $A$-Modul werde mit $\mathcal{N}(M)$ bezeichnet (vgl. Borewicz-Šafarevič [2], S. 142). $\mathcal{N}(M)$ ist gebrochenes $A$-Ideal, das i.a. nicht mit der Körpernorm $N_{K / k}(M)$ übereinstimmt; bei dieser lautet die zweite Bedingung $\alpha_{1} A \oplus$ $\alpha_{2} A \supset B$. 
Sind $M=\beta_{1} A \oplus \beta_{2} A$ und $\mathcal{O}(M)=\alpha_{1} A \oplus \alpha_{2} A$ freie $A$-Moduln und $\beta_{i}=a_{i 1} \alpha_{1}+a_{i 2} \alpha_{2}, a_{i j} \in k$, so ist $\mathcal{N}(M)=\operatorname{det}\left(a_{i j}\right) A$. Für jedes Primideal $\mathfrak{p}$ von $A$ gilt $\mathcal{N}(M)_{\mathfrak{p}}=\mathcal{N}\left(M_{\mathfrak{p}}\right)$. Der konjugierte Modul $M^{\prime}:=\left\{\alpha^{\prime} \mid \alpha \in M\right\}$ ist wieder $A$-Netz; es gilt $\mathcal{O}\left(M^{\prime}\right)=\mathcal{O}(M)^{\prime}=\mathcal{O}(M)$. Ist $\mathfrak{a} \neq 0$ gebrochenes $A$-Ideal, so folgt wegen seiner Invertierbarkeit $\mathcal{O}(\mathfrak{a} M)=\mathcal{O}(M)$.

Analog zu Lemma 1 in Borewicz-Šafarevič [2], S. 153, beweist man

Lemma 2.5. Für $\gamma \in K \backslash k, M:=A \oplus A \gamma$, sei

$$
\operatorname{Irr}(\gamma, k)=X^{2}+\frac{b}{a} X+\frac{c}{a} \quad(a, b, c \in A)
$$

das Minimalpolynom von $\gamma$ über $k$ und

$$
\mathfrak{a}:=A \cap \frac{a}{b} A \cap \frac{a}{c} A=g g T(b A, c A)^{-1} k g V(g g T(b A, c A), a A) .
$$

Dann ist $\mathcal{O}(M)=A \oplus \mathfrak{a} \gamma$.

Sind $\mathcal{O}_{1}, \mathcal{O}_{2} \subset K A$-Ordnungen und $\mathfrak{a} \neq 0$ gebrochenes $A$-Ideal mit $\mathfrak{a} \mathcal{O}_{1}=\mathcal{O}_{2}$, so ist $\mathcal{O}_{1}=\mathfrak{a}^{-1} \mathcal{O}_{2}=\mathfrak{a}^{-1} \mathcal{O}_{2} \mathcal{O}_{2}=\mathcal{O}_{1} \mathcal{O}_{2}=\mathcal{O}_{1} \mathfrak{a} \mathcal{O}_{1}=\mathfrak{a} \mathcal{O}_{1}=\mathcal{O}_{2}$. Damit und mit Lemma 2.5 kann folgendes Lemma genauso bewiesen werden wie (8), S. 155 und Satz 2, S. 156 in [2], falls $A$ ein Hauptidealring ist. Der allgemeine Fall wird mit (5) auf diesen Spezialfall zurückgeführt.

Lemma 2.6. (a) $M M^{\prime}=\mathcal{N}(M) \mathcal{O}(M)$ für A-Netze $M \subset K$.

(b) Für jede A-Ordnung $\mathcal{O} \subset K$ ist $G(\mathcal{O}):=\{M \mid M \subset K$ A-Netz, $\mathcal{O}(M)=\mathcal{O}\}$ bezüglich Modulmultiplikation eine abelsche Gruppe. Insbesondere ist $G(B)$ die Gruppe der gebrochenen B-Ideale.

Mit den Bezeichnungen (6) und (8) sei die $A$-Ordnung

$$
\mathcal{O}:=A \oplus \mathfrak{b} \mathfrak{c} \omega \subset B
$$

definiert. Durch Lokalisieren folgt

$$
N_{K / k}(\mathcal{O})=\mathfrak{c} .
$$

Lemma 2.7. $\phi: G(\mathcal{O}) \rightarrow G(B), M \rightarrow M B$, ist surjektiver Gruppenhomomorphismus.

Beweis. 1. Aus der Invertierbarkeit von Elementen aus $G(\mathcal{O})$ folgt, daß $\phi$ wohldefinierter Gruppenhomomorphismus ist.

2. Sei $A$ zunächst Hauptidealring, $\mathfrak{b}=t A, \mathfrak{c}=r A$. Um die Surjektivität von $\phi$ nachzuweisen, genügt es zu zeigen, daß jedes $N=A \oplus A \gamma \in G(B)$ $(\gamma \in K \backslash k)$ unter $\phi$ ein Urbild hat. Sei

$$
\operatorname{Irr}(\gamma, k)=X^{2}+\frac{b}{a} X+\frac{c}{a}, \quad a, b, c \in A,(a, b, c)=1 .
$$

Indem man $\gamma$ durch $\gamma+g$,

$$
g:=\prod_{p \in A \text { prim: } p \mid a, p \nmid c} p \in A,
$$


ersetzt, kann o.E. $(a, c)=1$ angenommen werden. Dann existieren $l, m \in A$ mit $r=l m,(l, m)=1,(l, a)=1,(m, c)=1$. Lemma 2.5 liefert für das $A$-Netz $M:=m A \oplus l \gamma A$

$$
\mathcal{O}(M)=\mathcal{O}\left(A \oplus \frac{l}{m} \gamma A\right)=A \oplus A a m^{2} \frac{l}{m} \gamma A=A+\operatorname{lm}(A+A a \gamma)
$$

und wegen $N \in G(B)$

$$
A \oplus A a \gamma=\mathcal{O}(N)=B=A \oplus t \omega A,
$$

d.h. insgesamt $M \in G(\mathcal{O})$. Aus (11) und $a \gamma^{2}=-b \gamma-c$ folgt $\phi(M)=N$.

3. Sei $A$ jetzt beliebig, $N \in G(B)$ und o.E. $N \subset B$. Die Menge

$$
\mathcal{M}:=\left\{\mathfrak{p} \mid \mathfrak{p} \neq 0 \text { Primideal in } A, \mathfrak{p} \mid \mathfrak{c} \text { oder } N_{\mathfrak{p}} \neq B_{\mathfrak{p}}\right\}
$$

ist endlich. Für $\mathfrak{p} \in \mathcal{M}$ existiert nach 2. ein $A_{\mathfrak{p}}$-Netz $M(\mathfrak{p}) \subset K$ mit $\mathcal{O}(M(\mathfrak{p}))=\mathcal{O}_{\mathfrak{p}}, M(\mathfrak{p}) B_{\mathfrak{p}}=N_{\mathfrak{p}}$. Der $A$-Modul $M:=\bigcap_{\mathfrak{p} \in \mathcal{M}} M(\mathfrak{p}) \cap B$ ist $A$-Netz, und es gilt

$$
M_{\mathfrak{p}}=M(\mathfrak{p}) \quad \text { für alle } \mathfrak{p} \in \mathcal{M} .
$$

Zum Beweis der nichttrivialen Inklusion sein $\alpha \in M(\mathfrak{p})$. Für $\mathfrak{q} \in \mathcal{M} \backslash\{\mathfrak{p}\}$ ist $A_{\mathfrak{q}}$ diskreter Bewertungsring und es gibt $n \geq 0$ mit

$$
\{a \in A \mid a \alpha \in M(\mathfrak{q})\}=A \cap\left\{a \in A_{\mathfrak{q}} \mid a \alpha \in M(\mathfrak{q})\right\}=A \cap\left(\mathfrak{q} A_{\mathfrak{q}}\right)^{n}=\mathfrak{q}^{n},
$$

d.h. es gibt $a_{\mathfrak{q}} \in A \backslash \mathfrak{p}$ mit $a_{\mathfrak{q}} \alpha \in M(\mathfrak{q})$. Ferner existiert wegen $M(\mathfrak{p}) \subset N_{\mathfrak{p}} \subset$ $B_{\mathfrak{p}}$ ein $s \in A \backslash \mathfrak{p}$ mit $s \alpha \in B$. Wegen $\left(\prod_{\mathfrak{p} \neq \mathfrak{q} \in \mathcal{M}} a_{\mathfrak{q}}\right) s \alpha \in M,\left(\prod_{\mathfrak{p} \neq \mathfrak{q} \in \mathcal{M}} a_{\mathfrak{q}}\right) s \in$ $A \backslash \mathfrak{p}$ ist $\alpha \in M_{\mathfrak{p}}$.

Analog beweist man $M_{\mathfrak{p}}=B_{\mathfrak{p}}$ für alle $\mathfrak{p} \notin \mathcal{M}$. Zusammen mit (12) folgt durch Lokalisieren $M B=N$ und $\mathcal{O}(M)=\mathcal{O}$.

Lemma 2.8. Für ein $A$-Netz $M \subset K$ gilt $\mathcal{O}(M)=\mathcal{O}, B M=M \Leftrightarrow$ Es gibt $\xi \in B$ mit $\mathfrak{c} B+\xi B=B, M=\mathfrak{c} B+\xi A$.

Beweis. " $\Leftarrow ": M B=B$ ist klar. Ist $\alpha \in \mathcal{O}(M)$, so ist $\alpha \xi \in M=$ $\mathfrak{c} B+\xi A$, d.h. es gibt $x \in A$ mit $(\alpha-x) \xi \in \mathfrak{c} B$. Da $\mathfrak{c} B$ und $\xi B$ teilerfremd sind, ist $\alpha-x \in \mathfrak{c} B$, d.h. $\alpha \in A+\mathfrak{c} B=\mathcal{O}$.

Für $\alpha \in \mathcal{O} \subset B$ ist ferner $\alpha M=\alpha \mathfrak{c} B+\alpha \xi A \subset \mathfrak{c} B+(A+\mathfrak{b} \mathfrak{c} \omega) \xi A=$ $\mathfrak{c} B+\xi A=M$, d.h. $\alpha \in \mathcal{O}(M)$.

" $\Rightarrow$ ": 1. Sei $A$ zunächst diskreter Bewertungsring mit Primelement $p$, $\mathfrak{b}=t A, \mathfrak{c}=r A$. Nach O'Meara [15], Theorem (81:3), existiert zu $M \subset B=$ $A \oplus t \omega A$ eine Zerlegung

$$
M=p^{e} A \oplus \delta A, \quad \delta=p^{f}+p^{g} \varepsilon t \omega, e, f, g \geq 0, \varepsilon \in E(A) .
$$

Wegen $B=M B=p^{e} B+\delta B$ sind $p^{e}$ und $\delta \delta^{\prime}$ in $A$ teilerfremd. $\delta / p^{e}$ ist Nullstelle von

$$
X^{2}-\frac{\left(\delta+\delta^{\prime}\right) p^{e}}{p^{2 e}} X+\frac{\delta \delta^{\prime}}{p^{2 e}},
$$


d.h. nach Lemma 2.5 ist

$$
A \oplus \operatorname{tr} \omega A=\mathcal{O}=\mathcal{O}(M)=A \oplus p^{2 e} \frac{\delta}{p^{e}} A=A \oplus p^{e}\left(p^{f}+p^{g} \varepsilon t \omega\right) A,
$$

d.h. $r A=p^{e+g} A$. Damit ist

$$
r B=p^{e+g} A \oplus p^{e+g} t \omega A \subset p^{e} A \oplus\left(p^{f}+p^{g} \varepsilon t \omega\right) A=M .
$$

Für $e=0$ sei $\xi:=1 \in M$, ansonsten $\xi:=\delta=p^{f}+p^{g} \varepsilon t \omega \in M$. Wegen $B=p^{e} B+\left(p^{f}+p^{g} \varepsilon t \omega\right) B$ ist ferner $\min \{e, f, g\}=0$. Damit folgt in jedem Fall $r B+\xi A=M$.

2. Sei $A$ jetzt beliebig. Ist $\mathfrak{p} \mid \mathfrak{c}$ Primideal von $A$, so existiert nach 1 . ein $\xi(\mathfrak{p}) \in B_{\mathfrak{p}}$ mit $M_{\mathfrak{p}}=\mathfrak{c} B_{\mathfrak{p}}+\xi(\mathfrak{p}) A_{\mathfrak{p}}$. Durch Multiplikation mit einem Element von $A \backslash \mathfrak{p}$ kann $\xi(\mathfrak{p}) \in B$ erreicht werden. Nach dem Chinesischen Restsatz existiert $\xi \in B$ mit $\xi \equiv \xi(\mathfrak{p}) \bmod \mathfrak{p}^{\operatorname{ord}_{\mathfrak{p}} \mathfrak{c}} B$ für alle $\mathfrak{p} \mid \mathfrak{c}$. Für diese $\mathfrak{p}$ ist dann $(\xi-\xi(\mathfrak{p})) A_{\mathfrak{p}} \subset \mathfrak{p}^{\operatorname{Ord} \mathfrak{p} \mathfrak{c}} B_{\mathfrak{p}}=(\mathfrak{c} B)_{\mathfrak{p}}$, d.h.

$$
M_{\mathfrak{p}}=\mathfrak{c} B_{\mathfrak{p}}+\xi(\mathfrak{p}) A_{\mathfrak{p}}+(\xi-\xi(\mathfrak{p})) A_{\mathfrak{p}}=(\mathfrak{c} B+\xi A)_{\mathfrak{p}} .
$$

Für $\mathfrak{p} \nmid \mathfrak{c}$ ist $\mathcal{O}\left(M_{\mathfrak{p}}\right)=\mathcal{O}_{\mathfrak{p}}=B_{\mathfrak{p}}, M_{\mathfrak{p}} B_{\mathfrak{p}}=B_{\mathfrak{p}}$, d.h. $\quad M_{\mathfrak{p}}=B_{\mathfrak{p}}=(\mathfrak{c} B)_{\mathfrak{p}}=$ $(\mathfrak{c} B+\xi A)_{\mathfrak{p}}$. Zusammen mit (13) ergibt sich $M=\mathfrak{c} B+\xi A$. Ferner ist $B=M B=\mathfrak{c} B+\xi B$.

Lemma 2.9. Für $A:=\mathcal{O}_{k}$ gilt

$$
|\operatorname{Kern} \phi|=N_{k / \mathbb{Q}}(\mathfrak{c}) \prod_{\mathfrak{p} \mid \mathfrak{c}}\left(1-\frac{\chi_{D}^{*}(\mathfrak{p})}{N_{k / \mathbb{Q}}(\mathfrak{p})}\right)=: \gamma(D) .
$$

B ew eis. Nach Lemma 2.8 ist

$$
\psi: E\left(\mathcal{O}_{K} / \mathfrak{c} \mathcal{O}_{K}\right) \rightarrow \operatorname{Kern} \phi, \quad \xi \bmod \mathfrak{c O}_{K} \rightarrow \mathfrak{c} \mathcal{O}_{K}+\xi \mathcal{O}_{k},
$$

wohldefinierter surjektiver Gruppenhomomorphismus. Er hat den Kern $E\left(\mathcal{O} / \mathfrak{c} \mathcal{O}_{K}\right)$. Der kanonische Homomorphismus

$$
\mathcal{O}_{k} \rightarrow \mathcal{O} / \mathfrak{c} \mathcal{O}_{K}, \quad x \rightarrow x \bmod \mathfrak{c}_{K}
$$

ist surjektiv und hat den Kern $\mathcal{O}_{k} \cap \mathfrak{c} \mathcal{O}_{K}=\mathfrak{c}$. Es gilt also

$$
|\operatorname{Kern} \phi|=\left|E\left(\mathcal{O}_{K} / \mathfrak{c} \mathcal{O}_{K}\right)\right|:|\operatorname{Kern} \psi|=\left|E\left(\mathcal{O}_{K} / \mathfrak{c} \mathcal{O}_{K}\right)\right|:\left|E\left(\mathcal{O}_{k} / \mathfrak{c}\right)\right| .
$$

Mit der Verallgemeinerung der Eulerschen $\phi$-Funktion (Narkiewicz [14], Theorem 1.8) und der Definition von $\chi_{D}^{*}$ folgt

$$
\begin{aligned}
\left|E\left(\mathcal{O}_{K} / \mathfrak{c} \mathcal{O}_{K}\right)\right| & =N_{K / \mathbb{Q}}\left(\mathfrak{c} \mathcal{O}_{K}\right) \prod_{\mathfrak{P} \mid \mathfrak{c} \mathcal{O}_{K}}\left(1-\frac{1}{N_{K / \mathbb{Q}}(\mathfrak{P})}\right) \\
& =N(\mathfrak{c})^{2} \prod_{\mathfrak{p} \mid \mathfrak{c}}\left(1-\frac{1}{N(\mathfrak{p})}\right)\left(1-\frac{\chi_{D}^{*}(\mathfrak{p})}{N(\mathfrak{p})}\right),
\end{aligned}
$$




$$
\left|E\left(\mathcal{O}_{k} / \mathfrak{c}\right)\right|=N(\mathfrak{c}) \prod_{\mathfrak{p} \mid \mathfrak{c}}\left(1-\frac{1}{N(\mathfrak{p})}\right)
$$

und damit aus (14) die Behauptung.

Wie bei Narkiewicz [14], Chap. III, §5, Bem. 4 der 1. Auflage von 1974 zeigt man, daß es eine Untergruppe $\mathcal{U} \subset k^{*}$ gibt, sodaß die direkte Zerlegung

$$
k^{*}=E\left(\mathcal{O}_{k}\right) \odot \mathcal{U}
$$

gilt.

Zwei $\mathcal{O}_{k}$-Netze $M_{1}, M_{2} \subset K$ heißen äquivalent im engen Sinn $\left(M_{1} \sim_{\text {eng }}\right.$ $M_{2}$ ), wenn es $\xi \in K^{*}$ gibt mit $N_{K / k}(\xi) \in \mathcal{U}$ und $M_{1}=\xi M_{2}$.

Sei $\nu(D):=\left[\left\{\alpha \in E\left(\mathcal{O}_{K}\right) \mid N_{K / k}(\alpha)=1\right\}:\left\{\alpha \in E(\mathcal{O}) \mid N_{K / k}(\alpha)=1\right\}\right]$.

Lemma 2.10. Für die in (2) definierte Gruppe gilt

$$
\begin{gathered}
U_{D}=\left\{\alpha \in E(\mathcal{O}) \mid N_{K / k}(\alpha)=1\right\}, \quad \nu(D)<\infty, \\
\left|G(\mathcal{O}) / \sim_{\text {eng }}\right|=\left|G\left(\mathcal{O}_{K}\right) / \sim_{\text {eng }}\right| \cdot \gamma(D): \nu(D) .
\end{gathered}
$$

B e w e is. Die Endlichkeit der Äquivalenzklassen-Anzahlen wird sich aus dem Beweis von Lemma 2.12 ergeben. Da $\mathcal{U}$ Gruppe ist, kann auf $\widetilde{G(\mathcal{O})}:=$ $G(\mathcal{O}) / \sim_{\text {eng }}$ und $G \widetilde{\left(\mathcal{O}_{K}\right)}:=G\left(\mathcal{O}_{K}\right) / \sim_{\text {eng }}$ vertreterweise die Multiplikation definiert werden. $\phi$ induziert einen Gruppenhomomorphismus

$$
\bar{\phi}: \widetilde{G(\mathcal{O})} \rightarrow \widetilde{G\left(\mathcal{O}_{K}\right)}, \quad[M]_{\mathrm{eng}} \rightarrow\left[M \mathcal{O}_{K}\right]_{\mathrm{eng}}
$$

der nach Lemma 2.7 surjektiv ist; $[M]_{\text {eng }}$ sei die Äquivalenzklasse von $M$.

Mit der Untergruppe $G:=\left\{\xi \mathcal{O}_{K} \mid \xi \in K^{*}, N_{K / k}(\xi) \in \mathcal{U}\right\} \subset G\left(\mathcal{O}_{K}\right)$ gilt dann unter Verwendung von $\mathcal{U} \cap E\left(\mathcal{O}_{k}\right)=\{1\}$,

$$
\widetilde{G(\mathcal{O})} /\left\{[M]_{\mathrm{eng}} \mid \phi(M) \in G\right\} \cong \widetilde{G\left(\mathcal{O}_{K}\right)},
$$

$\operatorname{Kern} \phi /\left\{\xi \mathcal{O} \mid \xi \in E\left(\mathcal{O}_{K}\right), N_{K / k}(\xi)=1\right\} \cong\left\{[M]_{\text {eng }} \mid \phi(M) \in G\right\}$,

$$
\begin{aligned}
\left\{\xi \in E\left(\mathcal{O}_{K}\right) \mid N_{K / k}(\xi)=1\right\} /\{\xi & \left.\in E(\mathcal{O}) \mid N_{K / k}(\xi)=1\right\} \\
& \cong\left\{\xi \mathcal{O} \mid \xi \in E\left(\mathcal{O}_{K}\right), N_{K / k}(\xi)=1\right\} .
\end{aligned}
$$

Mit Lemma 2.9 folgen daraus die beiden letzten Behauptungen.

Ist $\alpha=(x+y \sqrt{D}) / 2 \in U_{D}$, so ist $\alpha+\alpha^{\prime}=x \in \mathcal{O}_{k}$ und $\alpha \alpha^{\prime}=$ $\left(x^{2}-y^{2} D\right) / 4=1 \in \mathcal{O}_{k}$. Daraus folgt $\alpha=z+t \omega \in \mathcal{O}_{K}=\mathcal{O}_{k} \oplus \mathfrak{b} \omega$ und mit (7) und (8), $y^{2} \mathfrak{c}^{2} \mathfrak{b}^{2}\left(\omega-\omega^{\prime}\right)^{2}=\left(\alpha-\alpha^{\prime}\right)^{2} \mathcal{O}_{k}=t^{2}\left(\omega-\omega^{\prime}\right)^{2} \mathcal{O}_{k}$, d.h. $t \in y \mathfrak{c b} \subset \mathfrak{c b}$ und damit $\alpha \in \mathcal{O}$. Ebenso folgt $\alpha^{\prime} \in \mathcal{O}$ und damit $\alpha \in E(\mathcal{O})$. Die umgekehrte Richtung wird analog bewiesen.

Sei $\mathcal{M}_{D}:=\{M \in G(\mathcal{O}) \mid M$ freier $A$-Modul $\}$. Jede Äquivalenzklasse aus $G(\mathcal{O})$, die $\mathcal{M}_{D}$ schneidet, liegt ganz in $\mathcal{M}_{D}$.

LEMma 2.11. Hat $k$ ungerade Klassenzahl $h_{k}$, so gilt

$$
|\widetilde{G(\mathcal{O})}|=h_{k}\left|\mathcal{M}_{D} / \sim_{\text {eng }}\right| \text {. }
$$


B ew e is. Bezüglich der durch

$M_{1} \approx M_{2}: \Leftrightarrow$ Es existiert ein Automorphismus $\varrho$ des $k$-Vektorraums $K$ mit $M_{1}=\varrho\left(M_{2}\right)$

auf $G(\mathcal{O})$ definierten Äquivalenzrelation ist $\mathcal{M}_{D}$ eine Äquivalenzklasse. Jede

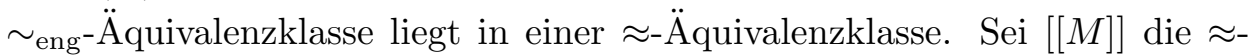
Äquivalenzklasse von $M$. Für $\mathfrak{a} \in G\left(\mathcal{O}_{k}\right)$ ist die Abbildung

$$
G(\mathcal{O}) \rightarrow G(\mathcal{O}), \quad M \rightarrow \mathfrak{a} M,
$$

wohldefiniert und bijektiv; Äquivalenzklassen bezüglich $\sim_{\text {eng }}$ und $\approx$ bleiben unter ihr erhalten, d.h.

$$
\sum_{[N]_{\text {eng }} \subset[[M]]} 1=\sum_{[P]_{\text {eng }} \subset[[\mathfrak{a} M]]} 1 \quad \text { für } M \in G(\mathcal{O}) .
$$

Sind $M_{1}, M_{2} \in G(\mathcal{O})$, so existieren nach Narkiewicz [14], Theorem 1.13, gebrochene Ideale $\mathfrak{a}_{1}, \mathfrak{a}_{2} \in G\left(\mathcal{O}_{k}\right)$ mit $M_{1} \cong \mathcal{O}_{k} \oplus \mathfrak{a}_{1}, M_{2} \cong \mathcal{O}_{k} \oplus \mathfrak{a}_{2}$, wobei $\cong$ die Isomorphie von $\mathcal{O}_{k}$-Moduln bezeichnet; sie kann jeweils zu einem Isomorphismus der $k$-Vektorräume $K$ und $k \oplus k$ fortgesetzt werden. Quadrieren ist auf der Idealklassengruppe von $k$ ein Isomorphismus, da sie ungerade Ordnung $h_{k}$ hat. Es gibt daher $\mathfrak{a} \in G\left(\mathcal{O}_{k}\right)$ und $0 \neq \alpha \in k$ mit $\alpha \mathfrak{a}^{2} \mathfrak{a}_{1}=\mathfrak{a}_{2}$. Wegen $\mathfrak{a} M_{1} \cong \mathfrak{a} \oplus \mathfrak{a} \mathfrak{a}_{1}$ folgt aus [14], Theorem 1.14, $\mathfrak{a} M_{1} \cong M_{2}$, d.h. $\mathfrak{a} M_{1} \approx M_{2}$ nach Fortsetzen des $\mathcal{O}_{k}$-Modul-Isomorphismus zu einem $k$-Automorphismus von $K$. Insgesamt gilt also

$$
\begin{gathered}
\sum_{[N]_{\text {eng }} \subset\left[\left[M_{1}\right]\right]} 1=\sum_{[P]_{\text {eng }} \subset\left[\left[M_{2}\right]\right]} 1, \\
|\widetilde{G(\mathcal{O})}|=\sum_{[[M]] \in G(\mathcal{O}) / \approx} 1 \sum_{[N]_{\text {eng }} \subset[[M]]} 1=|G(\mathcal{O}) / \approx| \cdot\left|\mathcal{M}_{D} / \sim_{\text {eng }}\right| .
\end{gathered}
$$

Sei $M_{0} \in G(\mathcal{O}), M_{0} \cong \mathcal{O}_{k} \oplus \mathfrak{a}_{0}, \mathfrak{a}_{0} \in G\left(\mathcal{O}_{k}\right)$. Für $\mathfrak{a}_{1}, \mathfrak{a}_{2} \in G\left(\mathcal{O}_{k}\right)$ folgt aus den oben zitierten Sätzen

$$
\left[\left[\mathfrak{a}_{1} M_{0}\right]\right]=\left[\left[\mathfrak{a}_{2} M_{0}\right]\right] \Leftrightarrow \mathfrak{a}_{1} \mathfrak{a}_{2}^{-1} \text { ist Hauptideal }
$$

und damit $|G(\mathcal{O}) / \approx|=h_{k}$.

LEMMA 2.12. Ist $h_{k}$ ungerade, so gilt

$$
h(D)=\frac{h_{K}}{h_{k}} \gamma(D) \frac{\left[E\left(\mathcal{O}_{k}\right): N_{K / k}\left(E\left(\mathcal{O}_{K}\right)\right)\right]}{\left[\left\{\alpha \in E\left(\mathcal{O}_{K}\right) \mid N_{K / k}(\alpha)=1\right\}: U_{D}\right]} .
$$

Beweis. Die folgenden Schlüsse sind analog zu denen in Zagier [29], S. 92ff.

(1) Für $M \in \mathcal{M}_{D}, M=\mathcal{O}_{k} \alpha \oplus \mathcal{O}_{k} \beta$ folgt aus

$$
\left|\begin{array}{cc}
\alpha & \alpha^{\prime} \\
\beta & \beta^{\prime}
\end{array}\right|^{2} \mathcal{O}_{k}=d_{K / k}(M)=N_{K / k}(M)^{2} d_{K / k}
$$


mit (8) und der Invarianz von $\left(\alpha \beta^{\prime}-\alpha^{\prime} \beta\right) / \sqrt{D}$ unter Konjugation

$$
\frac{\alpha \beta^{\prime}-\alpha^{\prime} \beta}{\sqrt{D}} \in k^{*} \quad \text { und } \quad \frac{\alpha \beta^{\prime}-\alpha^{\prime} \beta}{\sqrt{D}} \mathcal{O}_{k}=N_{K / k}(M) \mathfrak{c}^{-1},
$$

d.h. es gibt genau ein $n(M) \in \mathcal{U}$ mit

$$
N_{K / k}(M) \mathfrak{c}^{-1}=n(M) \mathcal{O}_{k}, \quad \frac{\alpha \beta^{\prime}-\alpha^{\prime} \beta}{n(M) \sqrt{D}} \in E\left(\mathcal{O}_{k}\right) .
$$

Die $\mathcal{O}_{k}$-Basis $(\alpha, \beta)$ von $M$ heißt positiv orientiert, falls $\alpha \beta^{\prime}-\alpha^{\prime} \beta=$ $n(M) \sqrt{D}$. Jedes $M \in \mathcal{M}_{D}$ hat eine solche Basis $(\alpha, \beta)$, und für sie wird definiert

$$
f_{M, \alpha, \beta}(x, y):=\frac{N_{K / k}(\alpha x+\beta y)}{n(M)}=\frac{\alpha \alpha^{\prime}}{n(M)} x^{2}+\frac{\alpha \beta^{\prime}+\alpha^{\prime} \beta}{n(M)} x y+\frac{\beta \beta^{\prime}}{n(M)} y^{2} .
$$

Wegen der positiven Orientiertheit der Basis hat diese quadratische Form die Diskriminante $D$. Man bezeichne ihre Koeffizienten mit $a, b, c$. Ist

$$
\operatorname{Irr}\left(\frac{\beta}{\alpha}, k\right)=X^{2}+\frac{r}{t} X+\frac{s}{t}, \quad r, s, t \in \mathcal{O}_{k},
$$

so folgt aus Lemma $2.5,(10)$ und (8)

$$
\begin{gathered}
\mathcal{O}=\mathcal{O}(M)=\mathcal{O}_{k} \oplus \mathfrak{a} \frac{\beta}{\alpha}, \quad \mathfrak{a}=g g T\left(r \mathcal{O}_{k}, s \mathcal{O}_{k}\right)^{-1} k g V\left(g g T\left(r \mathcal{O}_{k}, s \mathcal{O}_{k}\right), t \mathcal{O}_{k}\right), \\
D \mathcal{O}_{k}=N_{K / k}(\mathcal{O})^{2} d_{K / k}=d_{K / k}(\mathcal{O})=\mathfrak{a}^{2} \frac{\left(\alpha \beta^{\prime}-\alpha^{\prime} \beta\right)^{2}}{\left(\alpha \alpha^{\prime}\right)^{2}}=\mathfrak{a}^{2} \frac{n(M)^{2} D}{\left(\alpha \alpha^{\prime}\right)^{2}}
\end{gathered}
$$

d.h. $\mathfrak{a}=a \mathcal{O}_{k}$. Damit ergibt sich

$$
\begin{aligned}
a \mathcal{O}_{k}+b \mathcal{O}_{k}+c \mathcal{O}_{k}=a\left(\mathcal{O}_{k}+\frac{r}{t}\right. & \left.\mathcal{O}_{k}+\frac{s}{t} \mathcal{O}_{k}\right) \\
& =\frac{a}{t} g g T\left(t \mathcal{O}_{k}, g g T\left(r \mathcal{O}_{k}, s \mathcal{O}_{k}\right)\right)=\mathcal{O}_{k} .
\end{aligned}
$$

$f_{M, \alpha, \beta}$ ist also primitive quadratische Form über $\mathcal{O}_{k}$ mit Diskriminante $D$.

(2) Seien $M_{i} \in \mathcal{M}_{D}, i=1,2$, im engeren Sinn äquivalent mit positiv orientierten Basen $\left(\alpha_{i}, \beta_{i}\right)$. Ist $\lambda \in K^{*}, N_{K / k}(\lambda) \in \mathcal{U}, M_{1}=\lambda M_{2}$, so existiert $\left(\begin{array}{ll}p & q \\ v & w\end{array}\right) \in \mathrm{GL}_{2}\left(\mathcal{O}_{k}\right)$ mit

$$
\left(\begin{array}{c}
\alpha_{1} \\
\beta_{1}
\end{array}\right)=\left(\begin{array}{cc}
p & q \\
v & w
\end{array}\right)\left(\begin{array}{cc}
\lambda & \alpha_{2} \\
\lambda & \beta_{2}
\end{array}\right) .
$$

Es folgt $n\left(M_{1}\right) \mathfrak{c}=N_{K / k}(\lambda) n\left(M_{2}\right) \mathfrak{c}$, d.h. $n\left(M_{1}\right)=N_{K / k}(\lambda) n\left(M_{2}\right)$. Damit ist $f_{M_{1}, \alpha_{1}, \beta_{1}}(x, y)=f_{M_{2}, \alpha_{2}, \beta_{2}}(p x+v y, q x+w y)$ und wegen der positiven Orientiertheit $n\left(M_{1}\right) \sqrt{D}=(p w-q v) n\left(M_{1}\right) \sqrt{D}$, d.h. $p w-q v=1$. Also sind $f_{M_{i}, \alpha_{i}, \beta_{i}}, i=1,2$, äquivalent. 
Ist $\mathcal{A}_{D}$ die Menge aller primitiven quadratischen Formen über $\mathcal{O}_{k}$ mit Diskriminante $D$, so ist also die Abbildung

$$
\Phi: \mathcal{M}_{D} / \sim_{\mathrm{eng}} \rightarrow \mathcal{A}_{D} / \sim, \quad\left[M_{1}\right]_{\mathrm{eng}} \rightarrow\left[f_{M_{1}, \alpha_{1}, \beta_{1}}\right],
$$

wohldefiniert.

(3) Die Äquivalenzklasse jedes Elements von

$$
\begin{array}{r}
\mathcal{A}_{D}^{*}:=\left\{a x^{2}+b x y+c y^{2} \mid a \mathcal{O}_{k}+b \mathcal{O}_{k}+c \mathcal{O}_{k}=\mathcal{O}_{k},\right. \\
b^{2}-4 a c=D, \\
\left.a \in N_{K / k}\left(K^{*}\right) \mathcal{U}\right\}
\end{array}
$$

ist stets ganz in $\mathcal{A}_{D}^{*}$ enthalten ( $a \neq 0$, da $D$ kein Quadrat ist), und es gilt

$\operatorname{Bild} \Phi=\mathcal{A}_{D}^{*} / \sim$.

Dabei ist die Inklusion " $\subset$ " trivial. Umgekehrt, sei

$$
f=a x^{2}+b x y+c y^{2} \in \mathcal{A}_{D}^{*}, \quad M:=\mathcal{O}_{k} \oplus \mathcal{O}_{k} \beta, \beta:=\frac{b-\sqrt{D}}{2 a} \in K \backslash k .
$$

Aus Lemma 2.5 folgt $\mathcal{O}(M)=\mathcal{O}_{k} \oplus a \beta \mathcal{O}_{k}$. Wegen $a \beta \in \mathcal{O}_{K}=\mathcal{O}_{k} \oplus \mathfrak{b} \omega$ ist $a \beta=x+y \omega, x \in \mathcal{O}_{k}, y \in \mathfrak{b}$. Aus (7) und (8) folgt $D \mathcal{O}_{k}=\left(a \beta^{\prime}-a \beta\right)^{2} \mathcal{O}_{k}=$ $D y^{2} \mathfrak{b}^{-2} \mathfrak{c}^{-2}$, d.h. $y \mathcal{O}_{k}=\mathfrak{b c}$. Daraus folgt $\mathcal{O}(M)=\mathcal{O}$, d.h. $M \in \mathcal{M}_{D}$.

Seien $\lambda \in K^{*}, z \in \mathcal{U}$, mit $a=N_{K / k}(\lambda) z^{-1}$. Man definiere $\alpha_{1}:=\lambda, \beta_{1}:=\lambda \beta$. Mit (16) folgt, daß $\left(\alpha_{1}, \beta_{1}\right)$ positiv orientierte $\mathcal{O}_{k}$-Basis von $M_{1}:=\lambda M \in \mathcal{M}_{D}$ ist mit $z=n\left(M_{1}\right)$. Ferner ist $f_{M_{1}, \alpha_{1}, \beta_{1}}=f$, d.h. $[f]=\Phi\left(\left[M_{1}\right]_{\mathrm{eng}}\right)$.

$\Phi$ ist injektiv. Dazu sei $f \in \mathcal{A}_{D}^{*}$ und $M_{1}$ wie oben. Hat $\widetilde{M} \in \mathcal{M}_{D}$ die positiv orientierte Basis $(\widetilde{\alpha}, \widetilde{\beta})$ und ist $f_{\widetilde{M}, \widetilde{\alpha}, \widetilde{\beta}}=f$, so ist

$$
\beta=\frac{\widetilde{\beta}}{\widetilde{\alpha}} \text { und } \widetilde{M}=\widetilde{\alpha}\left(\mathcal{O}_{k} \oplus \beta \mathcal{O}_{k}\right)=\frac{\widetilde{\alpha}}{\lambda} M_{1} \text { mit } N_{K / k}\left(\frac{\widetilde{\alpha}}{\lambda}\right)=\frac{n(\widetilde{M})}{z} \in \mathcal{U}
$$

d.h. $\widetilde{M} \sim_{\text {eng }} M_{1}$. Ist nun $\widetilde{M} \in \mathcal{M}_{D}$ mit positiv orientierter Basis $(\widetilde{\widetilde{\alpha}}, \widetilde{\widetilde{\beta}})$ und $\Phi\left([\widetilde{M}]_{\text {eng }}\right)=[f]$, so existiert $\left(\begin{array}{cc}p & q \\ v & w\end{array}\right) \in \mathrm{SL}_{2}\left(\mathcal{O}_{k}\right)$ mit $f=f_{\widetilde{M}, \widetilde{\alpha}, \widetilde{\beta}} \widetilde{\widetilde{\beta}}(p x+q y, v x+$ $w y)=f_{\widetilde{M}, \widetilde{\alpha}, \widetilde{\beta}} ;$ dabei ist

$$
\left(\begin{array}{l}
\widetilde{\alpha} \\
\widetilde{\beta}
\end{array}\right):=\left(\begin{array}{ll}
p & v \\
q & w
\end{array}\right)\left(\begin{array}{c}
\widetilde{\widetilde{\alpha}} \\
\widetilde{\widetilde{\beta}}
\end{array}\right)
$$

positiv orientierte Basis von $\widetilde{M}$, d.h. nach dem eben Bewiesenen $\widetilde{M} \sim_{\text {eng }} M_{1}$.

Insgesamt ist

$$
\left|\mathcal{M}_{D} / \sim_{\text {eng }}\right|=\left|\mathcal{A}_{D}^{*} / \sim\right|
$$

(4) Durch

$f_{1}=a_{1} x^{2}+b_{1} x y+c_{1} y^{2} \approx f_{2}=a_{2} x^{2}+b_{2} x y+c_{2} y^{2}: \Leftrightarrow a_{1} / a_{2} \in N_{K / k}\left(K^{*}\right) \mathcal{U}$ ist auf $\mathcal{A}_{D}$ eine Äquivalenzrelation erklärt mit $f_{1} \sim f_{2} \Rightarrow f_{1} \approx f_{2}$. 
Sei $\mathcal{L} \in \mathcal{A}_{D} / \approx$ und $f=e^{-1} z x^{2}+\ldots \in \mathcal{L}, e \in E\left(\mathcal{O}_{k}\right), z \in \mathcal{U}$. Dann ist die Abbildung

$$
\mathcal{L} \rightarrow \mathcal{A}_{D e^{2}}^{*}, \quad g \rightarrow e g,
$$

bijektiv und mit der $\sim$ - Äquivalenz quadratischer Formen verträglich. Wegen $\mathcal{M}_{D e^{2}}=\mathcal{M}_{D}$ ist also nach (17)

$$
\begin{aligned}
|\mathcal{L} / \sim|=\left|\mathcal{A}_{D e^{2}}^{*} / \sim\right|=\left|\mathcal{M}_{D} / \sim_{\text {eng }}\right|, & \\
& \text { d.h. }\left|\mathcal{A}_{D} / \sim\right|=\left|\mathcal{A}_{D} / \approx\right| \cdot\left|\mathcal{M}_{D} / \sim_{\text {eng }}\right| .
\end{aligned}
$$

Die Abbildung

$$
\mathcal{A}_{D} / \approx \rightarrow k^{*} / N_{K / k}\left(K^{*}\right) \mathcal{U}, \quad f=a x^{2}+\ldots \rightarrow a \bmod N_{K / k}\left(K^{*}\right) \mathcal{U},
$$

ist bijektiv, da $e x^{2}+u x y+\frac{u^{2}-D}{4 e} y^{2}, e \in E\left(\mathcal{O}_{k}\right), u^{2} \equiv D(4)$, auf $e \bmod$ $N_{K / k}\left(K^{*}\right) \mathcal{U}$ abgebildet wird. Aus (18) folgt

$$
h(D)=\left|\mathcal{A}_{D} / \sim\right|=\left|\mathcal{M}_{D} / \sim_{\text {eng }}\right| \cdot\left[k^{*}: N_{K / k}\left(K^{*}\right) \mathcal{U}\right] .
$$

(5) Für den Gruppenhomomorphismus $N_{K / k}: E\left(\mathcal{O}_{K}\right) \rightarrow E\left(\mathcal{O}_{k}\right)$ gilt wegen der endlichen Erzeugtheit von $E\left(\mathcal{O}_{k}\right)$

$$
\begin{gathered}
E\left(\mathcal{O}_{k}\right)^{2} \subset \operatorname{Bild} N_{K / k} \subset E\left(\mathcal{O}_{k}\right), \\
m:=\left[E\left(\mathcal{O}_{k}\right): \operatorname{Bild} N_{K / k}\right] \leq\left[E\left(\mathcal{O}_{k}\right): E\left(\mathcal{O}_{k}\right)^{2}\right]<\infty, \\
E\left(\mathcal{O}_{k}\right) \rightarrow k^{*} / \mathcal{U} \operatorname{Bild} N_{K / k}, \quad a \rightarrow a \bmod \mathcal{U} \operatorname{Bild} N_{K / k},
\end{gathered}
$$

ist surjektiver Gruppenhomomorphismus, der Bild $N_{K / k}$ als Kern hat. Sei $v:=\left[N_{K / k}\left(K^{*}\right) \mathcal{U}:\right.$ Bild $\left.N_{K / k} \mathcal{U}\right]$. Dann ist also

(21) $\left[k^{*}: \mathcal{U} N_{K / k}\left(K^{*}\right)\right]=\left[E\left(\mathcal{O}_{k}\right): \operatorname{Bild} N_{K / k}\right]:\left[\mathcal{U} N_{K / k}\left(K^{*}\right): \mathcal{U} \operatorname{Bild} N_{K / k}\right]$

$$
=m / v<\infty \text {. }
$$

Ist $N_{K / k}\left(\alpha_{i}\right), \alpha_{i} \in K^{*}, i=1, \ldots, v$, vollständiges Repräsentantensystem von $N_{K / k}\left(K^{*}\right) \mathcal{U} /$ Bild $N_{K / k} \mathcal{U}, \mathcal{K} \subset G\left(\mathcal{O}_{K}\right)$ Äquivalenzklasse im weiteren Sinn, $\mathcal{A} \in \mathcal{K}$, so ist $\alpha_{i} \mathcal{A}, i=1, \ldots, v$, vollständiges Repräsentantensystem von $\mathcal{K} / \sim_{\text {eng }}$, d.h. $\left|\mathcal{K} / \sim_{\text {eng }}\right|=v$. Ist $h_{K}$ die Idealklassenzahl von $K \mathrm{im}$ weiteren Sinn, so gilt also

$$
\left|G\left(\mathcal{O}_{K}\right) / \sim_{\text {eng }}\right|=v h_{K} .
$$

Aus (19), (21), (22), Lemma 2.10 und Lemma 2.11 folgt schließlich

$$
h(D)=\left|\mathcal{M}_{D} / \sim_{\text {eng }}\right| \frac{m}{v}=\frac{|\widetilde{G(\mathcal{O})}|}{h_{k}} \frac{m}{v}=\frac{m}{\nu(D)} \frac{h_{K}}{h_{k}} \gamma(D) .
$$

Beweis von Proposition 2.4. Die Numerierung der Körperhomomorphismen sei wie folgt:

$$
\sigma_{1}(D)>0, \ldots, \sigma_{r_{1}}(D)>0, \sigma_{r_{1}+1}(D)<0, \ldots, \sigma_{r}(D)<0, \quad 0 \leq r_{1} \leq r .
$$


Die Vorzeichen der Wurzeln seien so gewählt, daß gilt

$$
\overline{\sqrt{\sigma_{i}(D)}}=\sqrt{\sigma_{i+s}(D)}, \quad r+1 \leq i \leq r+s .
$$

Für $\alpha=x+y \sqrt{D} \in K, x, y \in k$, sei

$$
\begin{aligned}
& \widetilde{\sigma}_{i}(\alpha):=\sigma_{i}(x)+\sigma_{i}(y) \sqrt{\sigma_{i}(D)}, \quad \widetilde{\widetilde{\sigma}}_{i}(\alpha):=\sigma_{i}(x)-\sigma_{i}(y) \sqrt{\sigma_{i}(D)}, \\
& 1 \leq i \leq r+2 s \text {. }
\end{aligned}
$$

Dann sind $\widetilde{\sigma}_{i}, \widetilde{\widetilde{\sigma}}_{i}$ für $1 \leq i \leq r_{1}$ die reellen und für $r_{1}+1 \leq i \leq r+2 s$ die komplexen $\mathbb{Q}$-Einbettungen von $K$ in $\mathbb{C}$. Sei $r^{\prime}:=2 r_{1}, 2 s^{\prime}:=2 n-2 r_{1}$. Es gilt

$$
\begin{aligned}
\overline{\widetilde{\sigma}}_{i} & =\widetilde{\widetilde{\sigma}}_{i} & & \text { für } r_{1}+1 \leq i \leq r, \\
\overline{\widetilde{\sigma}}_{i} & =\widetilde{\sigma}_{i+s} & & \text { für } r+1 \leq i \leq r+s, \\
\overline{\widetilde{\sigma}}_{i} & =\widetilde{\widetilde{\sigma}}_{i+s} & & \text { für } r+1 \leq i \leq r+s, \\
\widetilde{\widetilde{\sigma}}_{i}(\alpha) & =\widetilde{\sigma}_{i}\left(\alpha^{\prime}\right) & & \text { für } 1 \leq i \leq r+2 s, \alpha \in K .
\end{aligned}
$$

Sei

$$
\left(\operatorname{Kern} N_{K / k}\right)^{*}:=\left\{\alpha \in E\left(\mathcal{O}_{K}\right) \mid \alpha^{m} \in \operatorname{Kern} N_{K / k} \text { für ein } m \geq 1\right\} .
$$

Die Torsionsfreiheit von $E\left(\mathcal{O}_{K}\right) /\left(\operatorname{Kern} N_{K / k}\right)^{*}$ und $T\left(\left(\operatorname{Kern} N_{K / k}\right)^{*}\right)=$ $T(E(\mathcal{O}))$ liefert mit dem Hauptsatz über endlich erzeugte abelsche Gruppen

$$
\begin{aligned}
& E\left(\mathcal{O}_{K}\right)=\left(\operatorname{Kern} N_{K / k}\right)^{*} \odot\left\langle\varepsilon_{1}\right\rangle \odot \ldots \odot\left\langle\varepsilon_{l}\right\rangle, \\
& \left(\operatorname{Kern} N_{K / k}\right)^{*}=T\left(E\left(\mathcal{O}_{K}\right)\right) \odot\left\langle\eta_{1}\right\rangle \odot \ldots \odot\left\langle\eta_{p}\right\rangle, \\
& E\left(\mathcal{O}_{K}\right)=T\left(E\left(\mathcal{O}_{K}\right)\right) \odot\left\langle\eta_{1}\right\rangle \odot \ldots \odot\left\langle\eta_{p}\right\rangle \odot\left\langle\varepsilon_{1}\right\rangle \odot \ldots \odot\left\langle\varepsilon_{l}\right\rangle .
\end{aligned}
$$

Der Dirichletsche Einheitensatz ergibt mit $E\left(\mathcal{O}_{K}\right) / \operatorname{Kern} N_{K / k} \cong$ Bild $N_{K / k}$ und (20)

$l=\operatorname{rang} E\left(\mathcal{O}_{K}\right) / \operatorname{Kern} N_{K / k}=\operatorname{rang} E\left(\mathcal{O}_{k}\right)=r+s-1, \quad p+l=r^{\prime}+s^{\prime}-1$,

$$
\text { d.h. } p=r_{1}+s \text {. }
$$

Für $1 \leq j \leq p$ und eine $\mathbb{Q}$-Einbettung $\sigma: K \rightarrow \mathbb{C}$ ist

$$
\left|\sigma\left(\eta_{j}\right) \sigma\left(\eta_{j}^{\prime}\right)\right|=1
$$

Mit (23) ergibt sich daraus

$$
\left|\widetilde{\sigma}_{i}\left(\eta_{j}\right)\right|=1 \quad \text { für } r_{1}+1 \leq i \leq r, 1 \leq j \leq p,
$$

(27) $\quad \log \left|\sigma_{i}\left(N_{K / k}\left(\varepsilon_{q}\right)\right)\right|=\log \left|\widetilde{\sigma}_{i}\left(\varepsilon_{q}\right)\right|^{2} \quad$ für $r_{1}+1 \leq i \leq r, 1 \leq q \leq l$.

Nach (23) besteht die Regulatormatrix von $K$ aus zwei Spalten, wobei in der ersten die Matrizen

$$
\begin{gathered}
A_{1}=\left(\log \left|\widetilde{\sigma}_{i}\left(\eta_{j}\right)\right|\right)_{\substack{1 \leq i \leq r_{1} \\
1 \leq j \leq p}}, \quad A_{2}=\left(\log \left|\widetilde{\sigma}_{i}\left(\eta_{j}\right)\right|^{2}\right)_{\substack{r_{1}+1 \leq i \leq r \\
1 \leq j \leq p}}, \\
A_{3}=\left(\log \left|\widetilde{\sigma}_{i}\left(\eta_{j}\right)\right|^{2}\right)_{\substack{r+1 \leq i \leq r+s \\
1 \leq j \leq p}},
\end{gathered}
$$




$$
A_{4}=\left(\log \left|\widetilde{\sigma}_{i}\left(\eta_{j}^{\prime}\right)\right|\right)_{\substack{1 \leq i \leq r_{1} \\ 1 \leq j \leq p}}, \quad A_{5}=\left(\log \left|\widetilde{\sigma}_{i}\left(\eta_{j}^{\prime}\right)\right|^{2}\right)_{\substack{r+1 \leq i \leq r+s-1 \\ 1 \leq j \leq p}}
$$

und in der zweiten die entsprechenden Matrizen mit $\varepsilon_{q}, 1 \leq q \leq l$, statt $\eta_{j}$, $1 \leq j \leq p$, vorkommen. Wegen (25), (26) und (27) ist

$$
A_{2}=0, \quad A_{1}+A_{4}=0, \quad\left(\text { erste } s-1 \text { Zeilen von } A_{3}\right)+A_{5}=0,
$$

d.h.

$$
R_{K}=R_{1} \cdot R_{2}
$$

mit

$$
\begin{aligned}
& R_{1}=\operatorname{abs} d e t^{t}\left(\left(\log \left|\widetilde{\sigma}_{i}\left(\eta_{j}\right)\right|\right)_{\substack{1 \leq i \leq r_{1} \\
1 \leq j \leq p}},\left(\log \left|\widetilde{\sigma}_{i}\left(\eta_{j}\right)\right|^{2}\right)_{\substack{r+1 \leq i \leq r+s \\
1 \leq j \leq p}}\right), \\
& R_{2}=\left|\operatorname{det}\left(\log \left|\sigma_{i}\left(N_{K / k}\left(\varepsilon_{q}\right)\right)\right|\right)_{1 \leq i, q \leq r+s-1}\right| \cdot 2^{s-1} .
\end{aligned}
$$

Aus (24) folgt

Bild $N_{K / k}=T\left(\operatorname{Bild} N_{K / k}\right) \odot\left\langle N_{K / k}\left(\varepsilon_{1}\right)\right\rangle \odot \ldots \odot\left\langle N_{K / k}\left(\varepsilon_{l}\right)\right\rangle$,

d.h. $R_{2}$ ist der Regulator von Bild $N_{K / k}$. Da der Regulator von $E\left(\mathcal{O}_{k}\right)$ auch der Regulator $R_{k}$ von $k$ ist, folgt

$$
\left[E\left(\mathcal{O}_{k}\right): \operatorname{Bild} N_{K / k}\right]=\left[T\left(E\left(\mathcal{O}_{k}\right)\right): T\left(\operatorname{Bild} N_{K / k}\right)\right] \frac{R_{2}}{R_{k}} .
$$

Da $\left(\operatorname{Kern} N_{K / k}\right)^{*} / \operatorname{Kern} N_{K / k}$ eine endlich erzeugte Torsionsgruppe ist, folgt mit Lemma 2.10

$$
p=\operatorname{rang}\left(\operatorname{Kern} N_{K / k}\right)^{*}=\operatorname{rang} U_{D}
$$

und die Existenz einer direkten Zerlegung

$$
U_{D}=T\left(U_{D}\right) \odot\left\langle\beta_{1}\right\rangle \odot \ldots \odot\left\langle\beta_{p}\right\rangle .
$$

Mit

$$
R(D):=\operatorname{abs} d e t^{t}\left(\left(\log \left|\widetilde{\sigma}_{i}\left(\beta_{j}\right)\right|\right)_{\substack{1 \leq i \leq r_{1} \\ 1 \leq j \leq p}}\left(\log \left|\widetilde{\sigma}_{i}\left(\beta_{j}\right)\right|^{2}\right)_{\substack{r+1 \leq i \leq r+s \\ 1 \leq j \leq p}}\right)
$$

und da $R_{1}$ Regulator von $\left(\operatorname{Kern} N_{K / k}\right)^{*}$ ist, gilt

$$
\left[\left(\operatorname{Kern} N_{K / k}\right)^{*}: U_{D}\right]=\left[T\left(E\left(\mathcal{O}_{K}\right)\right): T\left(U_{D}\right)\right] \frac{R(D)}{R_{1}} .
$$

Einschränkung von $N_{K / k}$ auf $\left(\operatorname{Kern} N_{K / k}\right)^{*}$ liefert

$$
\left(\operatorname{Kern} N_{K / k}\right)^{*} / \operatorname{Kern} N_{K / k} \cong T\left(\operatorname{Bild} N_{K / k}\right)
$$

und damit

$$
R(D)=R_{1} \frac{\left|T\left(U_{D}\right)\right| \cdot\left[\operatorname{Kern} N_{K / k}: U_{D}\right]\left|T\left(\operatorname{Bild} N_{K / k}\right)\right|}{\left|T\left(E\left(\mathcal{O}_{K}\right)\right)\right|} .
$$


Mit der Klassenzahlformel für Zahlkörper ([2], S. 336, Satz 2) folgt aus Lemma 2.12, (28), (29), (31), Lemma 2.2, (8), Lemma 2.9, und Lemma 2.1

$$
\begin{aligned}
h(D)= & \frac{\left[E\left(\mathcal{O}_{k}\right): \operatorname{Bild} N_{K / k}\right]}{\left[\operatorname{Kern} N_{K / k}: U_{D}\right]} \gamma(D) \frac{\operatorname{Res}_{s=1} \zeta_{K}(s)\left|T\left(E\left(\mathcal{O}_{K}\right)\right)\right|\left|d_{K / \mathbb{Q}}\right|^{1 / 2}}{2^{r^{\prime}+s^{\prime}} \pi^{s^{\prime}} R_{K}} \\
& \times \frac{2^{r+s} \pi^{s} R_{k}}{\operatorname{Res}_{s=1} \zeta_{k}(s)\left|T\left(E\left(\mathcal{O}_{k}\right)\right)\right|\left|d_{k / \mathbb{Q}}\right|^{1 / 2}} \\
= & \frac{\left|T\left(U_{D}\right)\right|\left|d_{k / \mathbb{Q}}\right|^{1 / 2}\left|N_{K / k}(D)\right|^{1 / 2}}{R(D) 2^{p} \pi^{n-p}} L\left(1, \chi_{D}\right) .
\end{aligned}
$$

Da $\chi_{D}^{*}$ und $\chi_{D}$ Idealklassencharaktere im engeren Sinn sind und keine Hauptcharaktere, sind $L\left(s, \chi_{D}^{*}\right)$ und $L\left(s, \chi_{D}\right)$ Heckesche $L$-Reihen und damit ganze Funktionen (Landau, [11], Satz LXIII).

3. Der Spezialfall $p=1$. Erfüllt $k$ die in Abschnitt 1 genannten schärferen Voraussetzungen, so ist für $D \in \widetilde{\mathcal{D}}$

$$
\begin{aligned}
r & =n, \quad r_{1}=1, \quad s=0, \quad p=1, \\
k, K \subset \mathbb{R}, \quad \widetilde{\sigma}_{1}=\mathrm{id}_{K}, \quad T\left(K^{*}\right) & =\{ \pm 1\} .
\end{aligned}
$$

Ferner ist

$$
U_{D}=\{ \pm 1\} \odot\left\langle\varepsilon_{D}\right\rangle, \quad R(D)=\log \varepsilon_{D},
$$

mit eindeutig bestimmtem $\varepsilon_{D}>1$. Für $\alpha=(u+v \sqrt{D}) / 2 \in U_{D}$ gilt

$$
1<\alpha \leq x \Leftrightarrow 2<u \leq x+x^{-1}, v \sqrt{D}>0 .
$$

Für $D, u, v \in \mathcal{O}_{k}, u^{2}-D v^{2}=4, u>2,2 \leq i \leq n$ gilt: $v \neq 0, D>0$ und

$$
\sigma_{i}(D)<0 \Leftrightarrow\left|\sigma_{i}(u)\right|<2 \Rightarrow D \text { kein Quadrat in } \mathcal{O}_{k} .
$$

Für die Halbgruppe $\mathcal{V}:=\mathcal{U} \cap \mathcal{O}_{k}$ gilt

$$
\mathcal{O}_{k} \backslash\{0\}=E\left(\mathcal{O}_{k}\right) \odot \mathcal{V} .
$$

Für $D_{1} \in \widetilde{\mathcal{D}}, K:=k\left(\sqrt{D_{1}}\right), x \geq 2$, definiere man

$$
\begin{aligned}
S_{K} & :=\left\{(D, u, v) \mid D \in \mathcal{O}_{k} \text { Diskriminante, } k(\sqrt{D})=K, u \in \mathcal{O}_{k},\right. \\
& \left.v \in \mathcal{V}, u^{2}-D v^{2}=4,2<\sigma_{1}(u) \leq x,\left|\sigma_{i}(u)\right|<2, i=2, \ldots, n\right\} .
\end{aligned}
$$

Dann ist für $x^{\prime}:=\left(x+\sqrt{x^{2}-4}\right) / 2$

$$
\left|S_{K}\right|=\sum_{\alpha \in \operatorname{Kern} N_{K / k}: 1<\alpha \leq x^{\prime} \text { oder } 1<\alpha^{-1} \leq x^{\prime}}\left|\left\{(D, u, v) \in S_{K} \mid \frac{1}{2}(u+v \sqrt{D})=\alpha\right\}\right| .
$$

Ist $\alpha$ fest, $\left(D_{1}, u_{1}, v_{1}\right) \in S_{K},\left(u_{1}+v_{1} \sqrt{D}\right) / 2=\alpha$, so ist der Summand wegen $(36)$

$$
\leq \sum_{(D, v) \in \mathcal{O}_{k} \times \mathcal{V}: u_{1}^{2}-4=D v^{2}} 1 \leq \tau_{2}\left(\left(u_{1}^{2}-4\right) \mathcal{O}_{k}\right) \ll N_{k / \mathbb{Q}}\left(\left(u_{1}^{2}-4\right) \mathcal{O}_{k}\right)^{\varepsilon} \ll x^{2 \varepsilon} .
$$


Nach Lemma 2.10 ist [Kern $\left.N_{K / k}: U_{D_{1}}\right]<\infty$, d.h. Kern $N_{K / k}=\{ \pm 1\} \odot\langle\varepsilon\rangle$, $\varepsilon=\left(y+z \sqrt{D_{1}}\right) / 2>1$. Dabei ist $y=\varepsilon+\varepsilon^{\prime} \in \mathcal{O}_{k}, y^{2}-D_{1} z^{2}=4 \varepsilon \varepsilon^{\prime}=4$, d.h. $z \sqrt{D_{1}}>0, y>2,\left|\sigma_{i}(y)\right|<2, i=2, \ldots, n$, und damit $\varepsilon \geq y / 2$.

Das Bild von $\mathcal{O}_{k}$ unter der Abbildung

$$
\phi: k \rightarrow \mathbb{R}^{n}, \quad a \rightarrow{ }^{t}\left(\sigma_{1}(a), \ldots, \sigma_{n}(a)\right),
$$

ist ein Gitter in $\mathbb{R}^{n}$. Daher ist $\varepsilon \geq \mu(k)>1$ und

$$
\left|S_{K}\right| \ll x^{2 \varepsilon} \sum_{\alpha \in \operatorname{Kern} N_{K / k}: 1<\alpha \leq x^{\prime}} 1 \ll x^{2 \varepsilon} \frac{\log x^{\prime}}{\log \mu(k)} \ll x^{3 \varepsilon} .
$$

Ferner ist $\varepsilon_{D_{1}}=\varepsilon^{l}$ für ein $l \in \mathbb{N}$ und damit

$$
\varepsilon_{D_{1}} \geq \mu(k)>1
$$

für alle $D_{1} \in \widetilde{\mathcal{D}}$. Für ein Ideal $\mathfrak{q} \neq 0$ in $\mathcal{O}_{k}$ definiere man

$$
T_{\mathfrak{q}}:=\left\{K \mid \text { Es gibt } D \in \widetilde{\mathcal{D}} \text { mit } K=k(\sqrt{D}), d_{K / k}=\mathfrak{q}\right\} .
$$

Für $K, K_{1} \in T_{\mathfrak{q}}$ existieren $D_{K}, D_{K_{1}} \in \widetilde{\mathcal{D}}$ mit $K=k\left(\sqrt{D_{K}}\right), K_{1}=k\left(\sqrt{D_{K_{1}}}\right)$, und nach (8) ganze Ideale $\mathfrak{c}_{K}, \mathfrak{c}_{K_{1}}$ mit $D_{K} \mathcal{O}_{k}=\mathfrak{q c}_{K}^{2}, D_{K_{1}} \mathcal{O}_{k}=\mathfrak{q c}_{K_{1}}^{2}$. Da $h_{k}$ ungerade ist, existiert $l_{K} \in k^{*}$ mit $D_{K} D_{K_{1}}^{-1} \mathcal{O}_{k}=\left(\mathfrak{c}_{k} \mathfrak{c}_{K_{1}}^{-1}\right)^{2}=l_{K}^{2} \mathcal{O}_{k}$, d.h. es gibt $e_{K} \in E\left(\mathcal{O}_{k}\right)$ mit $D_{K}=e_{K} l_{K}^{2} D_{K_{1}}$. Für festes $K_{1}$ ist die Abbildung

$$
T_{\mathfrak{q}} \rightarrow E\left(\mathcal{O}_{k}\right) / E\left(\mathcal{O}_{k}\right)^{2}, \quad K \rightarrow e_{K} \bmod E\left(\mathcal{O}_{k}\right)^{2},
$$

injektiv. Damit gilt

$$
\left|T_{\mathfrak{q}}\right| \leq\left[E\left(\mathcal{O}_{k}\right): E\left(\mathcal{O}_{k}\right)^{2}\right] \ll 1 .
$$

4. Beweis von Satz 1.1. Für $x>1$ gilt mit (34) einerseits

(42) $\widetilde{S}_{m}(x):=\sum_{D \in \mathcal{D}, \alpha \in U_{D}: 1<\alpha \leq x}\left(h(D) \log \varepsilon_{D}\right)^{m}$

$$
=\sum_{D \in \mathcal{D}, u, v \in \mathcal{O}_{k}: u^{2}-D v^{2}=4,2<u \leq x+x^{-1}, v \sqrt{D}>0}\left(h(D) \log \varepsilon_{D}\right)^{m}
$$

und mit (33) andererseits

$$
\widetilde{S}_{m}(x)=\sum_{D \in \mathcal{D}, l \in \mathbb{N}: \varepsilon_{D}^{l} \leq x}\left(h(D) \log \varepsilon_{D}\right)^{m}=\sum_{l \geq 1} \Theta_{m}\left(x^{1 / l}\right) .
$$

Die Summe

$$
S_{m}(x):=\sum_{D \in \mathcal{O}_{k} \text { Diskr., } u \in \mathcal{O}_{k}, v \in \mathcal{V}:} \sum_{\substack{u^{2}-D v^{2}=4, N(v) \leq 2^{n-1} x \\ 2<\sigma_{1}(u) \leq x,\left|\sigma_{i}(u)\right|<2, i=2, \ldots, n}}\left(h(D) \log \varepsilon_{D}\right)^{m}
$$

ist wegen (35) wohldefiniert; wegen (36) und da $\phi\left(\mathcal{O}_{k}\right) \subset \mathbb{R}^{n}$ Gitter ist (siehe $(38))$, ist $S_{m}(x)$ endlich. 
Seien $\left(D_{j}, u_{j}, v_{j}\right), 1 \leq j \leq N$, die Tripel, über die in $S_{m}\left(x+x^{-1}\right)$ summiert wird. Wegen (35) ist $D_{j} \in \widetilde{\mathcal{D}}$ und es gibt genau ein $e_{j} \in E\left(\mathcal{O}_{k}\right)$ mit $e_{j}^{2} D_{j} \in \mathcal{D}, e_{j}^{-1} v_{j} \sqrt{e_{j}^{2} D_{j}}>0$. Dann sind $\left(e_{j}^{2} D_{j}, u_{j}, e_{j}^{-1} v_{j}\right), 1 \leq j \leq N$, genau die verschiedenen Tripel, über die in (42) summiert wird, d.h. es gilt

$$
\begin{aligned}
\widetilde{S}_{m}(x) & =\sum_{j=1}^{N}\left(h\left(e_{j}^{2} D_{j}\right) \log \varepsilon_{e_{j}^{2} D_{j}}\right)^{m} \\
& =\sum_{j=1}^{N}\left(h\left(D_{j}\right) \log \varepsilon_{D_{j}}\right)^{m}=S_{m}\left(x+x^{-1}\right) .
\end{aligned}
$$

Seien $D_{j} \bmod 4 \mathcal{O}_{k}, 1 \leq j \leq L$, die Quadrate in $\mathcal{O}_{k} / 4 \mathcal{O}_{k}$. Für $1 \leq j \leq L$, $0 \neq v \in \mathcal{O}_{k}$, seien $m_{i j}(v) \bmod 4 v^{2}, 1 \leq i \leq s_{j}(v)$, die Lösungen von $x^{2} \equiv$ $4+D_{j} v^{2}\left(4 v^{2}\right)$.

Mit dem Chinesischen Restsatz und $(x-2) \mathcal{O}_{k}+(x+2) \mathcal{O}_{k} \mid 4 \mathcal{O}_{k}$ folgt

$$
\begin{aligned}
s_{j}(v) & \leq N_{k / \mathbb{Q}}\left(4 \mathcal{O}_{k}\right) \cdot\left|\left\{x \bmod v^{2} \mid x^{2} \equiv 4\left(v^{2}\right)\right\}\right| \\
& \ll \prod_{\mathfrak{p} \mid v}\left|\left\{x \bmod \mathfrak{p}^{2} \operatorname{ord}_{\mathfrak{p} v} \mid x \equiv \pm 2\left(\mathfrak{p}^{2 \max \left\{\operatorname{ord}_{\mathfrak{p}} v-\operatorname{ord}_{\mathfrak{p}} 2,0\right\}}\right)\right\}\right| \\
& \leq \prod_{\mathfrak{p} \mid v} 2 N_{k / \mathbb{Q}}(\mathfrak{p})^{2 \operatorname{ord}_{\mathfrak{p}} v-2 \max \left\{\operatorname{ord}_{\mathfrak{p}} v-\operatorname{ord}_{\mathfrak{p}} 2,0\right\}} \leq 2^{\omega\left(v \mathcal{O}_{k}\right)} N_{k / \mathbb{Q}}\left(4 \mathcal{O}_{k}\right),
\end{aligned}
$$

wobei $\omega(\mathfrak{a}):=\sum_{\mathfrak{p} \mid \mathfrak{a}} 1$ ist. Mit $\tau_{l}(\mathfrak{a}):=\sum_{\mathfrak{a}_{1} \ldots \mathfrak{a}_{l}=\mathfrak{a}} 1$ gilt wie im rationalen Fall

$$
2^{\omega(\mathfrak{a})} \leq \tau_{2}(\mathfrak{a}) \ll N(\mathfrak{a})^{\varepsilon}, \quad s_{j}(v) \ll N(v)^{\varepsilon} .
$$

Sei $f_{v, i, j}(t):=16 v^{2} t^{2}+8 m_{i j} t+\left(m_{i j}^{2}-4\right) / v^{2} \in \mathcal{O}_{k}[t]$. Dann gilt für $0 \neq v \in$ $\mathcal{O}_{k}, 1 \leq j \leq L, u \in \mathcal{O}_{k}$,

$$
\begin{aligned}
u^{2}-D v^{2}=4 \text { lösbar mit einem } D \equiv D_{j}(4) \\
\Leftrightarrow \text { Es gibt } 1 \leq i \leq s_{j}(v) \text { mit } u \equiv m_{i j}\left(4 v^{2}\right) .
\end{aligned}
$$

Lemma 4.1. Für jedes Primideal $\mathfrak{p} \nmid 2$ zerfällt $f_{v, i, j} \bmod \mathfrak{p}$ im algebraischen Abschluß von $\mathcal{O}_{k} / \mathfrak{p}$ in ein oder zwei verschiedene Linearfaktoren; insbesondere ist es nicht das Nullpolynom modp.

B e w e is. Wegen $\mathfrak{p} \nmid m_{i j} \mathcal{O}_{k}+v \mathcal{O}_{k}$ hat $f_{v, i, j}^{\prime} \bmod \mathfrak{p}$ keine oder eine Nullstelle, die nicht Nullstelle von $f_{v, i, j} \bmod \mathfrak{p}$ ist.

Für ein ganzes Ideal $\mathfrak{a} \neq 0$ von $\mathcal{O}_{k}$ sei $\mathcal{M}(\mathfrak{a})$ vollständiges Repräsentantensystem von $\mathcal{O}_{k} / 4 \mathcal{O}_{k}$. Für $0 \neq v \in \mathcal{O}_{k}, 1 \leq j \leq L$, sei

$$
c_{j}(v, \mathfrak{a}):=\sum_{i=1}^{s_{j}(v)} \sum_{a \in \mathcal{M}(\mathfrak{a})}\left(\frac{f_{v, i, j}(a)}{\mathfrak{a}}\right)
$$


((4) ist für beliebiges $D \in \mathcal{O}_{k}$ sinnvoll). Wegen $f_{v, i, j}(a) \equiv\left(m_{i j}^{2}-4\right) / v^{2} \equiv$ $D_{j}(4)$ ist $f_{v, i, j}(a)$ immer Diskriminante. Die Definition ist unabhängig von der speziellen Wahl von $\mathcal{M}(\mathfrak{a})$, da das Gewicht $4 \mathfrak{a}$-periodisch ist.

Lemma 4.2. Für ein Primideal $\mathfrak{p} \nmid 2$ und einen Charakter $\phi$ von $\left(\mathcal{O}_{k} / \mathfrak{p},+\right)$ gilt

$$
\left|\sum_{a \bmod \mathfrak{p}}\left(\frac{f_{v, i, j}(a)}{\mathfrak{p}}\right) \phi(a)\right| \leq 2 N_{k / \mathbb{Q}}(\mathfrak{p})^{1 / 2} .
$$

B ew eis. $(\dot{\bar{p}})$ ist Charakter von $\left(\left(\mathcal{O}_{k} / \mathfrak{p}\right)^{*}, \cdot\right)($ Hecke [5], S. 196) und nicht der Hauptcharakter. Aus den Weilschen Charaktersummenabschätzungen (Schmidt [19], Lemma 2C, S.11 und Theorem 2C und 2G, S. 43 und 45) zusammen mit Lemma 4.1 folgt die Behauptung.

Für ein ganzes Ideal $\mathfrak{a} \neq 0$ definiere man $K(\mathfrak{a}):=\prod_{\mathfrak{p} \mid \mathfrak{a}: \operatorname{ord}_{\mathfrak{p}} \mathfrak{a} \text { ungerade }} \mathfrak{p}$.

Lemma 4.3. Sei $0 \neq v \in \mathcal{O}_{k}, \mathfrak{a} \neq 0$ Ideal von $\mathcal{O}_{k}, 1 \leq j \leq L, 1 \leq i \leq$ $s_{j}(v), \mathcal{R}$ vollständiges Repräsentantensystem von $\mathcal{O}_{k} / 4 \mathfrak{a}, \phi$ Charakter von $\left(\mathcal{O}_{k} / 4 \mathfrak{a},+\right)$. Dann ist

$$
\sum_{a \in \mathcal{R}}\left(\frac{f_{v, i, j}(a)}{\mathfrak{a}}\right) \phi(a) \ll N_{k / \mathbb{Q}}(\mathfrak{a}) N_{k / \mathbb{Q}}(K(\mathfrak{a}))^{-1 / 2} 2^{\omega(\mathfrak{a})} .
$$

Beweis. Seien $4 \mathfrak{a}=\mathfrak{p}_{1}^{a_{1}} \ldots \mathfrak{p}_{t}^{a_{t}}, \mathfrak{a}=\mathfrak{p}_{1}^{c_{1}} \ldots \mathfrak{p}_{t}^{c_{t}}$ Primidealzerlegungen. Nach dem Chinesischen Restsatz existieren die Isomorphismen der folgenden Gruppe und ihrer Dualgruppe:

$$
\mathcal{O}_{k} / 4 \mathfrak{a} \cong \mathcal{O}_{k} / \mathfrak{p}_{1}^{a_{1}} \oplus \ldots \oplus \mathcal{O}_{k} / \mathfrak{p}_{t}^{a_{t}}, \quad\left(\widehat{\mathcal{O}_{k} / 4} \mathfrak{a}\right) \cong\left(\widehat{\mathcal{O}_{k} / \mathfrak{p}_{1}^{a_{1}}}\right) \odot \ldots \odot\left(\widehat{\mathcal{O}_{k} / \mathfrak{p}_{t}^{a_{t}}}\right)
$$

Die abzuschätzende Summe $S$ ist unabhängig von $\mathcal{R}$ und damit

$$
\begin{aligned}
S & =\sum_{b_{r} \bmod \mathfrak{p}_{r}^{a_{r}, r=1, \ldots, t}} \prod_{l=1}^{t}\left(\frac{f_{v, i, j}\left(b_{l}\right)}{\mathfrak{p}_{l}}\right)^{c_{l}} \phi_{l}\left(b_{l}\right) \\
& =\prod_{l=1}^{t} \sum_{b_{l}}\left(\frac{f_{v, i, j}\left(b_{l}\right)}{\mathfrak{p o d}_{l}}\right)^{a_{l}}{ }^{c_{l}} \phi_{l}\left(b_{l}\right)=: \prod_{l=1}^{t} S_{l}
\end{aligned}
$$

wobei $\phi_{l}$ der durch $\phi$ auf $\mathcal{O}_{k} / \mathfrak{p}_{l}^{a_{l}}$ induzierte additive Charakter ist. Trivialerweise ist

$$
\left|S_{l}\right| \leq \sum_{b_{l} \bmod \mathfrak{p}_{l}^{a_{l}}} 1=N_{k / \mathbb{Q}}\left(\mathfrak{p}_{l}^{a_{l}}\right) .
$$

Ist $\mathfrak{p}_{l} \nmid 2, c_{l}$ ungerade, so ist $a_{l}=c_{l}$ und

$$
S_{l}=\sum_{d \bmod \mathfrak{p}_{l}} \sum_{c \in \mathfrak{p}_{l} / \mathfrak{p}_{l}^{c_{l}}}\left(\frac{f_{v, i, j}(d+c)}{\mathfrak{p}_{l}}\right) \phi_{l}(d+c)
$$




$$
=\sum_{d \bmod \mathfrak{p}_{l}}\left(\frac{f_{v, i, j}(d)}{\mathfrak{p}_{l}}\right) \phi_{l}(d) \sum_{c \in \mathfrak{p}_{l} / \mathfrak{p}_{l}^{c_{l}}} \phi_{l}(c) .
$$

Ist $\phi_{l}$ auf der Untergruppe $\mathfrak{p}_{l} / \mathfrak{p}_{l}^{c_{l}} \subset \mathcal{O}_{k} / \mathfrak{p}_{l}^{c_{l}}$ nicht der Hauptcharakter, so verschwindet die zweite Summe und es ist $S_{l}=0$. Im anderen Fall kann $\phi_{l}$ als Charakter von $\mathcal{O}_{k} / \mathfrak{p}_{l}$ aufgefaßt werden, und mit Lemma 4.2 folgt $\left|S_{l}\right| \leq N_{k / \mathbb{Q}}\left(\mathfrak{p}_{l}^{c_{l}-1}\right) \cdot 2 N_{k / \mathbb{Q}}\left(\mathfrak{p}_{l}\right)^{1 / 2}$. Aus (48) und (49) folgt damit

$$
\begin{aligned}
|S| & \leq \prod_{l: \mathfrak{p}_{l} \mid 2 \text { oder } c_{l} \text { gerade }} N_{k / \mathbb{Q}}\left(\mathfrak{p}_{l}\right)^{a_{l}} \prod_{l: \mathfrak{p}_{l} \nmid 2, c_{l} \text { ungerade }} 2 N_{k / \mathbb{Q}}\left(\mathfrak{p}_{l}\right)^{a_{l}-1 / 2} \\
& \ll N_{k / \mathbb{Q}}(\mathfrak{a}) N_{k / \mathbb{Q}}(K(\mathfrak{a}))^{-1 / 2} 2^{\omega(\mathfrak{a})} .
\end{aligned}
$$

Sei $\alpha_{1}, \ldots, \alpha_{n}$ eine feste $\mathbb{Z}$-Basis von $\mathcal{O}_{k}$.

Lemma 4.4. Sei $0 \neq v \in \mathcal{O}_{k}, \mathfrak{a} \neq 0$ ganzes Ideal, $1 \leq j \leq L, 1 \leq i \leq$ $s_{j}(v)$. Dann existiert eine $\mathbb{Z}$-Basis $\beta_{1}, \ldots, \beta_{n}$ von $4 \mathfrak{a}$ mit $\beta_{l}=a_{l 1} \alpha_{1}+\ldots+$ $a_{l l} \alpha_{l}, a_{l q} \in \mathbb{Z}, l=1, \ldots, n$. Für $0 \leq u_{l} \leq v_{l} \leq\left|a_{l l}\right|-1, l=1, \ldots, n$, $e \in E\left(\mathcal{O}_{k}\right)$, gilt

$$
\begin{aligned}
& \sum_{u_{l} \leq t_{l} \leq v_{l}, l=1, \ldots, n}\left(\frac{f_{v, i, j}\left(e \sum_{l=1}^{n} \alpha_{l} t_{l}\right)}{\mathfrak{a}}\right) \\
& \ll N(\mathfrak{a}) N(K(\mathfrak{a}))^{-1 / 2} 2^{\omega(\mathfrak{a})} \log ^{n} N(4 \mathfrak{a}) .
\end{aligned}
$$

B eweis. Die Existenz der Basis $\beta_{1}, \ldots, \beta_{n}$ folgt aus Theorem 35 von Hecke [5]. Nach Theorem 36 in [5] ist $N(4 \mathfrak{a})=\left|a_{11} \ldots a_{n n}\right|$ und

$$
\mathcal{R}:=\left\{e \sum_{l=1}^{n} \alpha_{l} x_{l}\left|x_{l} \in \mathbb{Z}, 0 \leq x_{l}<\right| a_{l l} \mid, l=1, \ldots, n\right\}
$$

vollständiges Repräsentantensystem von $\mathcal{O}_{k} / 4 \mathfrak{a}$. Seien $\alpha_{1}^{*}, \ldots, \alpha_{n}^{*}$ und $\beta_{1}^{*}, \ldots$ $\ldots, \beta_{n}^{*}$ die dualen Basen zu $\alpha_{1}, \ldots, \alpha_{n}$ und $\beta_{1}, \ldots, \beta_{n}$. Dann sind $\mathcal{O}_{k}^{*}=$ $\alpha_{1}^{*} \mathbb{Z} \oplus \ldots \oplus \alpha_{n}^{*} \mathbb{Z}$ und $(4 \mathfrak{a})^{*}=\beta_{1}^{*} \mathbb{Z} \oplus \ldots \oplus \beta_{n}^{*} \mathbb{Z}$ die dualen Moduln zu $\mathcal{O}_{k}$ und $4 \mathfrak{a}$ (Lang [12], S. 57, Proposition 1). Ist $\beta_{l}^{*}=\sum_{m=1}^{n} b_{l m} \alpha_{m}^{*}, b_{l m} \in \mathbb{Q}$, so gilt

$$
\begin{aligned}
\delta_{l t}=\operatorname{Sp}_{k / \mathbb{Q}}\left(\beta_{l}^{*} \beta_{t}\right)=\sum_{m=1}^{n} \sum_{u=1}^{n} b_{l m} a_{t u} \operatorname{Sp}_{k / \mathbb{Q}}\left(\alpha_{m}^{*} \alpha_{u}\right)=\sum_{m=1}^{n} b_{l m} a_{t m}, \\
1 \leq l, t \leq n .
\end{aligned}
$$

Da $\left(a_{l m}\right)$ untere Dreiecksmatrix ist, ist also $\left(b_{l m}\right)$ obere Dreiecksmatrix, d.h.

$$
b_{l m}=0 \quad \text { für } l>m, \quad b_{l l}=a_{l l}^{-1} \quad \text { für alle } l .
$$

Für $x_{1}, \ldots, x_{n} \in \mathbb{Z}$ bzw. $\lambda_{1}, \ldots, \lambda_{n} \in \mathbb{Z}$ sei

$$
F\left(x_{1}, \ldots, x_{n}\right):=\left(\frac{f_{v, i, j}\left(e \sum_{l=1}^{n} \alpha_{l} x_{l}\right)}{\mathfrak{a}}\right)
$$




$$
\begin{aligned}
\widehat{F}\left(\lambda_{1}, \ldots, \lambda_{n}\right) & :=\sum_{0 \leq x_{l}<\left|a_{l l}\right|, l=1, \ldots, n} F\left(x_{1}, \ldots, x_{n}\right) e\left(\sum_{m, r=1}^{n} b_{m r} \lambda_{m} x_{r}\right) \\
& =\sum_{0 \leq x_{l}<\left|a_{l l}\right|, l=1, \ldots, n} F\left(x_{1}, \ldots, x_{n}\right) e\left(\operatorname{Sp}_{k / \mathbb{Q}}\left(\gamma \sum_{l=1}^{n} e \alpha_{l} x_{l}\right)\right), \\
\gamma & =\gamma\left(\lambda_{1}, \ldots, \lambda_{n} ; \mathfrak{a}\right):=e^{-1} \sum_{m=1}^{n} \beta_{m}^{*} \lambda_{m} \in(4 \mathfrak{a})^{*} .
\end{aligned}
$$

Dabei ist $e(x):=e^{2 \pi i x} . \phi(\alpha):=e\left(\operatorname{Sp}_{k / \mathbb{Q}}(\gamma \alpha)\right)$ ist Charakter von $\left(\mathcal{O}_{k} / 4 \mathfrak{a},+\right)$. Mit (50) folgt aus Lemma 4.3 für alle $\lambda_{1}, \ldots, \lambda_{n} \in \mathbb{Z}$

$$
\widehat{F}\left(\lambda_{1}, \ldots, \lambda_{n}\right)=\sum_{\alpha \in \mathcal{R}}\left(\frac{f_{v, i, j}(\alpha)}{\mathfrak{a}}\right) \phi(\alpha) \ll N(\mathfrak{a}) N(K(\mathfrak{a}))^{-1 / 2} 2^{\omega(\mathfrak{a})} .
$$

Aus (51) und der Orthogonalitätsrelation folgt durch Auswerten der Summen von innen nach außen

$$
\begin{aligned}
& \sum_{0 \leq \lambda_{1}<\left|a_{11}\right|} \ldots \sum_{0 \leq \lambda_{n}<\left|a_{n n}\right|} e\left(\sum_{m, r=1}^{n} b_{m r} \lambda_{m}\left(x_{r}-t_{r}\right)\right) \\
&= \begin{cases}\left|a_{11} \ldots a_{n n}\right| & \text { für }\left(x_{1}, \ldots, x_{n}\right)=\left(t_{1}, \ldots, t_{n}\right), \\
0 & \text { sonst }\end{cases}
\end{aligned}
$$

für $0 \leq t_{l}, x_{l}<\left|a_{l l}\right|, t_{l}, x_{l} \in \mathbb{Z}, l=1, \ldots, n$. Damit gilt für $0 \leq t_{l}<\left|a_{l l}\right|$, $t_{l} \in \mathbb{Z}, l=1, \ldots, n$,

$$
\begin{aligned}
& F\left(t_{1}, \ldots, t_{n}\right) \\
= & \left|a_{11} \ldots a_{n n}\right|^{-1} \sum_{0 \leq \lambda_{l}<\left|a_{l l}\right|, l=1, \ldots, n} \widehat{F}\left(\lambda_{1}, \ldots, \lambda_{n}\right) e\left(-\sum_{m, r=1}^{n} b_{m r} \lambda_{m} t_{r}\right) .
\end{aligned}
$$

Seien $u_{l}, v_{l}$ wie in der Voraussetzung, $\lambda_{1}, \ldots, \lambda_{n} \in \mathbb{Z}$. Mit (51) und $\|x\|:=$ $\min _{l \in \mathbb{Z}}|x-l|$ gilt

$$
\begin{aligned}
\left|G\left(\lambda_{1}, \ldots, \lambda_{n}\right)\right| & :=\left|\sum_{u_{l} \leq t_{l} \leq v_{l}, l=1, \ldots, n} e\left(-\sum_{m, r=1}^{n} b_{m r} \lambda_{m} t_{r}\right)\right| \\
& =\prod_{r=1}^{n}\left|\sum_{u_{r} \leq t_{r} \leq v_{r}} e\left(\left(-\sum_{m=1}^{n} b_{m r} \lambda_{m}\right) t_{r}\right)\right| \\
& \leq \prod_{r=1}^{n} \min \left\{\left|a_{r r}\right|, \frac{1}{2}\left\|\sum_{m=1}^{r} b_{m r} \lambda_{m}\right\|^{-1}\right\} .
\end{aligned}
$$

Summation über $\lambda_{n}$ liefert für $\lambda_{1}, \ldots, \lambda_{n-1} \in \mathbb{Z}$ 


$$
\begin{aligned}
\sum_{0 \leq \lambda_{n}<\left|a_{n n}\right|} \mid & G\left(\lambda_{1}, \ldots, \lambda_{n-1}, \lambda_{n}\right) \mid \\
\ll & \prod_{r=1}^{n-1} \min \left\{\left|a_{r r}\right|,\left.|| \sum_{m=1}^{r} b_{m r} \lambda_{m}\right|^{-1}\right\} \\
& \times \sum_{0 \leq \lambda_{n}<\left|a_{n n}\right|} \min \left\{\left|a_{n n}\right|,\left\|\sum_{m=1}^{n-1} b_{m n} \lambda_{m}+a_{n n}^{-1} \lambda_{n}\right\|^{-1}\right\} \\
\ll & \prod_{r=1}^{n-1} \min \{\} \cdot 2\left(\left|a_{n n}\right|+\sum_{1 \leq l \leq \frac{1}{2}\left|a_{n n}\right|} \frac{1}{l /\left|a_{n n}\right|}\right) \\
\ll & \prod_{r=1}^{n-1} \min \{\}\left|a_{n n}\right| \log \left(\left|a_{n n}\right|+2\right)
\end{aligned}
$$

mit «-Konstante unabhängig von $b_{m n}$ und $\lambda_{1}, \ldots, \lambda_{n-1}$. $(n-1)$-fache Wiederholung dieses Schlusses liefert

$$
\sum_{0 \leq \lambda_{l}<\left|a_{l l}\right|, l=1, \ldots, n}\left|G\left(\lambda_{1}, \ldots, \lambda_{n}\right)\right| \ll \prod_{l=1}^{n}\left|a_{l l}\right| \log \left(\left|a_{l l}\right|+2\right) .
$$

Damit folgt aus (53) und (52)

$$
\begin{aligned}
& \sum_{\substack{u_{l} \leq t_{l} \leq v_{l}, l=1, \ldots, n \\
=}} F\left(t_{1}, \ldots, t_{n}\right) \\
&\left|a_{11} \ldots a_{n n}\right|^{-1} \sum_{0 \leq \lambda_{l}<\left|a_{l l}\right|, l=1, \ldots, n} \widehat{F}\left(\lambda_{1}, \ldots, \lambda_{n}\right) G\left(\lambda_{1}, \ldots, \lambda_{n}\right) \\
& \ll\left|a_{11} \ldots a_{n n}\right|^{-1} N(\mathfrak{a}) N(K(\mathfrak{a}))^{-1 / 2} 2^{\omega(\mathfrak{a})} \sum_{0 \leq \lambda_{l}<\left|a_{l l}\right|, l=1, \ldots, n}\left|G\left(\lambda_{1}, \ldots, \lambda_{n}\right)\right| \\
& \ll N(\mathfrak{a}) N(K(\mathfrak{a}))^{-1 / 2} 2^{\omega(\mathfrak{a})} \log ^{n} N(4 \mathfrak{a}) .
\end{aligned}
$$

LeMma 4.5. Für jedes Gitter $\Gamma \subset \mathbb{R}^{n-1}$ existiert eine Konstante $c>0$ mit der Eigenschaft: Für alle $x={ }^{t}\left(x_{1}, \ldots, x_{n-1}\right) \in \mathbb{R}^{n-1}$ gibt es $y \in \Gamma$ mit $x_{i}-c \leq y_{i} \leq x_{i}+c, i=1, \ldots, n-1$.

B ew eis. Wähle für $2 c$ die Kantenlänge eines achsenparallelen Würfels mit Mittelpunkt 0, der ein Fundamentalparallelotop von $\Gamma$ enthält.

Lemma 4.6. Ist $G \subset \mathbb{R}^{n}$ Gitter mit Basis $z_{1}, \ldots, z_{n}$ und Fundamentalparallelotop

$$
\mathcal{F}:=\left\{\sum_{l=1}^{n} \lambda_{l} z_{l} \mid 0 \leq \lambda_{l}<1, \lambda_{l} \in \mathbb{R}\right\}
$$


und $H \subset \mathbb{R}^{n}$ Hyperebene, so existiert $1 \leq l_{0} \leq n$ mit der Eigenschaft

$$
\left|\left\{\lambda \in \mathbb{Z} \mid\left(\gamma+\lambda z_{l_{0}}+\mathcal{F}\right) \cap H \neq \emptyset\right\}\right| \leq n \quad \text { für alle } \gamma \in G .
$$

B eweis. Angenommen, die Behauptung ist falsch. Zu jedem $1 \leq l \leq n$ existieren dann $\gamma_{l} \in G, \mu_{l} \in \mathbb{Z},\left|\mu_{l}\right| \geq n, u_{l}, u_{l}^{\prime} \in \mathcal{F}$ mit $\gamma_{l}+u_{l}, \gamma_{l}+$ $\mu_{l} z_{l}+u_{l}^{\prime} \in H$. Ist $\widetilde{H}$ die zu $H$ parallele Hyperebene durch 0 , so ist also $x_{l}:=\mu_{l} z_{l}+\left(u_{l}^{\prime}-u_{l}\right) \in \widetilde{H}$. Für $x \in \mathbb{R}^{n}$ bezeichne $x^{(j)}$ die $j$-te Koordinate von $x$ in der Basis $z_{1}, \ldots, z_{n}$. Nach Definition von $\mathcal{F}$ ist $\left|u_{l}^{(j)}-u_{l}^{\prime(j)}\right|<1$ für alle $j=1, \ldots, n$, d.h.

$$
\left|x_{l}^{(l)}\right|=\left|\mu_{l}+\left(u_{l}^{\prime}-u_{l}\right)^{(l)}\right|>n-1, \quad\left|x_{l}^{(j)}\right|=\left|\left(u_{l}^{\prime}-u_{l}\right)^{(j)}\right|<1
$$

Da $x_{1}, \ldots, x_{n} \operatorname{im}(n-1)$-dimensionalen affinen Teilraum $\widetilde{H}$ liegen, existiert $0 \neq\left(b_{1}, \ldots, b_{n}\right) \in \mathbb{R}^{n}$ mit $\sum_{l=1}^{n} b_{l} x_{l}=0$. Nimmt man o.E. $\left|b_{1}\right| \geq\left|b_{2}\right|, \ldots,\left|b_{n}\right|$ an, so ist $b_{1} \neq 0$ und aus $\sum_{l=1}^{n} b_{l} x_{l}^{(1)}=0$ folgt mit (55) der Widerspruch

$$
\left|b_{1}\right|(n-1)<\left|b_{1} x_{1}^{(1)}\right|=\left|\sum_{l=2}^{n} b_{l} x_{l}^{(1)}\right| \leq \sum_{l=2}^{n}\left|b_{l}\right|\left|x_{l}^{(1)}\right| \leq\left|b_{1}\right|(n-1) .
$$

Lemma 4.7. Für $0 \neq v \in \mathcal{O}_{k}, \mathfrak{a} \neq 0$ ganzes Ideal, $1 \leq j \leq L, 1 \leq i \leq$ $s_{j}(v), A_{l}<B_{l}, l=1, \ldots, n, V:=\prod_{l=1}^{n}\left(B_{l}-A_{l}\right)$ gilt

$$
\begin{aligned}
T:= & \sum_{\substack{a \in \mathcal{O}_{k}:{ }^{2} \\
A_{l}<\sigma_{l}(a) \leq B_{1} \\
A_{l}(a)<B_{l}, l=2, \ldots, n}}\left(\frac{f_{v, i, j}(a)}{\mathfrak{a}}\right) \\
= & \left.\sum_{a \in \mathcal{M}(\mathfrak{a})}\left(\frac{f_{v, i, j}(a)}{\mathfrak{a}}\right)\right) \frac{V}{N(4 \mathfrak{a})\left|d_{k / \mathbb{Q}}\right|^{1 / 2}} \\
& +O\left(\left(V^{(n-1) / n}+1\right) N(\mathfrak{a}) N(K(\mathfrak{a}))^{-1 / 2} 2^{\omega(\mathfrak{a})} \log ^{n} N(4 \mathfrak{a})\right), \\
& \sum_{a \in \mathcal{M}(\mathfrak{a})}\left(\frac{f_{v, i, j}(a)}{\mathfrak{a}}\right) \ll_{k} N(\mathfrak{a}) N(K(\mathfrak{a}))^{-1 / 2} 2^{\omega(\mathfrak{a})} .
\end{aligned}
$$

B ew eis. Mit (38) ist $\Gamma:=\phi\left(\mathcal{O}_{k}\right) \subset \mathbb{R}^{n}$ Gitter und $\Gamma^{\prime}:=\phi(4 \mathfrak{a})$ Untergitter von $\Gamma$. Man definiere die Abbildung

$$
\psi: E\left(\mathcal{O}_{k}\right) \rightarrow \mathbb{R}^{n-1}, \quad a \rightarrow{ }^{t}\left(\log \left|\sigma_{1}(a)\right|, \ldots, \log \left|\sigma_{n-1}(a)\right|\right) .
$$

Nach dem Dirichletschen Einheitensatz ist $\psi\left(E\left(\mathcal{O}_{k}\right)\right)$ Gitter in $\mathbb{R}^{n-1}$. Nach Lemma 4.5 existiert dazu eine Konstante $c=c(k)>0$, sodaß $e \in E\left(\mathcal{O}_{k}\right)$ existiert mit

$$
\begin{array}{r}
-\log \left(B_{l}-A_{l}\right)+\log V^{1 / n}-c \leq \log \left|\sigma_{l}(e)\right| \leq-\log \left(B_{l}-A_{l}\right)+\log V^{1 / n}+c, \\
l=1, \ldots, n-1 .
\end{array}
$$


Für

$$
\left(C_{l}, D_{l}\right):=\left\{\begin{array}{ll}
\left(A_{l} \sigma_{l}(e), B_{l} \sigma_{l}(e)\right), & \text { falls } \sigma_{l}(e)>0, \\
\left(B_{l} \sigma_{l}(e), A_{l} \sigma_{l}(e)\right), & \text { falls } \sigma_{l}(e)<0,
\end{array} \quad l=1, \ldots, n,\right.
$$

gilt damit

$$
\begin{gathered}
e^{-c} V^{1 / n} \leq\left(B_{l}-A_{l}\right)\left|\sigma_{l}(e)\right|=D_{l}-C_{l} \leq e^{c} V^{1 / n}, \quad l=1, \ldots, n-1, \\
\prod_{l=1}^{n}\left(D_{l}-C_{l}\right)=V, \\
e^{-c(n-1)} V^{1 / n} \leq D_{n}-C_{n}=V \prod_{l=1}^{n-1}\left(D_{l}-C_{l}\right)^{-1} \leq e^{c(n-1)} V^{1 / n} .
\end{gathered}
$$

Im Folgenden wird der Fall $\sigma_{1}(e)>0$ behandelt. Man setze

$$
\Phi(x):=\left(\frac{f_{v, i, j}\left(e^{-1} \phi^{-1}(x)\right)}{\mathfrak{a}}\right) \quad \text { für } x \in \Gamma .
$$

$x_{l}:=\phi\left(\alpha_{l}\right), l=1, \ldots, n$, ist Basis von $\Gamma$ und $y_{l}:=\phi\left(\beta_{l}\right), l=1, \ldots, n$, ist Basis von $\Gamma^{\prime}$ mit $y_{l}=a_{l 1} x_{1}+\ldots+a_{l l} x_{l}, l=1, \ldots, n$. Sei

$$
\begin{gathered}
\mathcal{F}:=\left\{\sum_{l=1}^{n} \mu_{l} x_{l}\left|0 \leq \mu_{l}<\right| a_{l l} \mid, \mu_{l} \in \mathbb{R}, l=1, \ldots, n\right\}, \\
Q:=\left(C_{1}, D_{1}\right] \times\left(C_{2}, D_{2}\right) \times \ldots \times\left(C_{n}, D_{n}\right) .
\end{gathered}
$$

Dann wird $\mathbb{R}^{n}$ durch die Mengen $y+\mathcal{F}, y \in \Gamma^{\prime}$, lückenlos und überschneidungsfrei überdeckt, und es gilt

$$
\begin{aligned}
\text { (56) } & T=\sum_{\substack{C_{1}<\sigma_{1}(a) \leq D_{1} \\
a \in \mathcal{O}_{k}:}}\left(\frac{f_{v, i, j}\left(e^{-1} a\right)}{\mathfrak{a}}\right)=\sum_{x \in \Gamma \cap Q} \Phi(x) \\
= & \sum_{y \in \Gamma^{\prime}: y+\mathcal{F} \subset \dot{Q}} \sum_{\substack{x \in \Gamma \cap(y+\mathcal{F}) \\
C_{l}(a)<D_{l}, l=2, \ldots, n}} \Phi(x)+\sum_{y \in \Gamma^{\prime}:(y+\mathcal{F}) \cap \partial Q \neq \emptyset} \sum_{x \in \Gamma \cap Q \cap(y+\mathcal{F})} \Phi(x) .
\end{aligned}
$$

$\partial Q$ bestehe aus den Hyperebenenflächenstücken $S_{l}, l=1, \ldots, 2 n$. Sei $H_{l}$ die Hyperebene, in der $S_{l}$ liegt. Der zweite Term von (56) hat dann den Wert

$$
\begin{gathered}
R=\sum_{l=1}^{2 n} R_{l}, \\
R_{l}:=\sum_{y \in \Gamma^{\prime}:(y+\mathcal{F}) \cap S_{l} \neq \emptyset,(y+\mathcal{F}) \cap\left(S_{1} \cup \ldots \cup S_{l-1}\right)=\emptyset} \sum_{x \in \Gamma \cap Q \cap(y+\mathcal{F})} \Phi(x) .
\end{gathered}
$$

Für festes $1 \leq l \leq 2 n$ existiert zum Gitter $G:=\bigoplus_{j=1}^{n} \mathbb{Z}\left|a_{j j}\right| x_{j}$ ein $1 \leq \iota \leq n$ mit

(58) $\quad\left|\left\{\lambda \in \mathbb{Z} \mid\left(\gamma+\lambda\left|a_{\iota \iota}\right| x_{\iota}+\mathcal{F}\right) \cap H_{l} \neq \emptyset\right\}\right| \leq n \quad$ für alle $\gamma \in G$. 
Bezeichnet $P$ die Projektion von $\mathbb{R}^{n}$ auf $\bigoplus_{m \neq \iota} \mathbb{R} x_{m}$ längs $x_{\iota}$ und setzt man $x=x^{\prime}+\mu_{\iota} x_{\iota}, x^{\prime}=P(x)$, so gilt

$$
\left|R_{l}\right| \leq \sum_{x^{\prime} \in P(\Gamma \cap Q)}\left|I\left(x^{\prime}\right)\right|
$$

mit $I\left(x^{\prime}\right)=I\left(x^{\prime}, \iota, l\right):=\sum_{\mu} \Phi\left(x^{\prime}+\mu x_{\iota}\right)$, wobei über alle $\mu \in \mathbb{Z}$ summiert wird, zu denen es ein $y \in \Gamma^{\prime} \operatorname{mit}(y+\mathcal{F}) \cap S_{l} \neq \emptyset,(y+\mathcal{F}) \cap\left(S_{1} \cup \ldots \cup S_{l-1}\right)=\emptyset$, $x^{\prime}+\mu x_{\iota} \in(y+\mathcal{F}) \cap Q$ gibt. Wegen $\max _{1 \leq r \leq n}\left(D_{r}-C_{r}\right) \ll V^{1 / n}$ und da $P$ lineare Abbildung ist, gilt

$$
|P(\Gamma \cap Q)| \leq\left|\left(\bigoplus_{m \neq \iota} \mathbb{Z} x_{m}\right) \cap P(Q)\right| \ll\left(V^{1 / n}+1\right)^{n-1} .
$$

Für $x^{\prime} \in \bigoplus_{m \neq \iota} \mathbb{Z} x_{m}, y \in \Gamma^{\prime}$, folgt aus der $\Gamma^{\prime}$-Periodizität von $\Phi(x)$

$$
\begin{aligned}
I\left(x^{\prime}, y\right) & :=\sum_{\mu \in \mathbb{Z}: x^{\prime}+\mu x_{\iota} \in(y+\mathcal{F}) \cap Q} \Phi\left(x^{\prime}+\mu x_{\iota}\right) \\
& =\sum_{\mu \in \mathbb{Z}:\left(x^{\prime}-y\right)+\mu x_{\iota} \in \mathcal{F} \cap(Q-y)} \Phi\left(\left(x^{\prime}-y\right)+\mu x_{\iota}\right) .
\end{aligned}
$$

Da $\mathcal{F} \cap(Q-y)$ konvex ist, existieren ganzzahlige $0 \leq u_{\iota} \leq v_{\iota}<\left|a_{\iota \iota}\right|$ und $0 \leq u_{m}<\left|a_{m m}\right|, m \neq \iota$, mit

$$
I\left(x^{\prime}, y\right)=\sum_{u_{\iota} \leq t_{\iota} \leq v_{\iota}, t_{m}=u_{m}, m \neq \iota} \Phi\left(\sum_{m=1}^{n} t_{m} x_{m}\right) .
$$

Mit Lemma 4.4 ist also $I\left(x^{\prime}, y\right) \ll N(\mathfrak{a}) N(K(\mathfrak{a}))^{-1 / 2} 2^{\omega(\mathfrak{a})} \log ^{n} N(4 \mathfrak{a})=: E$. Damit ist

$$
I\left(x^{\prime}\right)=\sum_{\substack{(y+\mathcal{F}) \cap S_{l} \neq \emptyset \\ y \in \Gamma^{\prime}:}} I\left(x^{\prime}, y\right) \ll E\left|M\left(x^{\prime}\right)\right|
$$

mit $M\left(x^{\prime}\right):=\left\{y \in \Gamma^{\prime} \mid(y+\mathcal{F}) \cap H_{l} \neq \emptyset\right.$, es gibt $\mu \in \mathbb{Z}$ mit $\left.x^{\prime}+\mu x_{\iota} \in(y+\mathcal{F})\right\}$.

Sei $x^{\prime}=\sum_{m \neq \iota}\left(\lambda_{m}\left|a_{m m}\right|+v_{m}\right) x_{m} \in P(\Gamma \cap Q), \lambda_{m}, v_{m} \in \mathbb{Z}, 0 \leq v_{m}<$ $\left|a_{m m}\right|$, fest. Ist $y \in M\left(x^{\prime}\right)$ und $\mu=\lambda_{\iota}\left|a_{\iota \iota}\right|+v_{\iota} \in \mathbb{Z}, \lambda_{\iota}, v_{\iota} \in \mathbb{Z}, 0 \leq v_{\iota}<\left|a_{\iota \iota}\right|$, ein Wert mit $x^{\prime}+\mu x_{\iota} \in y+\mathcal{F}$, so ist

$$
y+\mathcal{F}=\left(y-x^{\prime}-\mu x_{\iota}\right)+\sum_{m=1}^{n} v_{m} x_{m}+\sum_{m=1}^{n} \lambda_{m}\left|a_{m m}\right| x_{m}+\mathcal{F},
$$

wobei der erste Summand in $-\mathcal{F}$ und der zweite in $\mathcal{F}$ liegt. Die $3^{n}$ NachbarParallelotope von $\mathcal{F}_{1}:=\mathcal{F}\left(\lambda_{1}, \ldots, \lambda_{n}\right):=\sum_{m=1}^{n} \lambda_{m}\left|a_{m m}\right| x_{m}+\mathcal{F}$ im Gitter $\bigoplus_{m=1}^{n} \mathbb{Z}\left|a_{m m}\right| x_{m}$ überdecken also $y+\mathcal{F}$. Wegen $y \in M\left(x^{\prime}\right)$ existiert ein Nachbar-Parallelotop $\mathcal{F}_{2}:=\mathcal{F}\left(\lambda_{1}+t_{1}, \ldots, \lambda_{n}+t_{n}\right),\left|t_{m}\right| \leq 1,1 \leq m \leq n$, von $\mathcal{F}_{1}$ mit $\mathcal{F}_{2} \cap(y+\mathcal{F}) \cap H_{l} \neq \emptyset$. Die Nachbar-Parallelotope von $\mathcal{F}_{2}$ 
überdecken deshalb wieder $y+\mathcal{F}$. Setzt man $\lambda:=\lambda_{\iota}+t_{\iota}$, so ist $y+\mathcal{F}$ enthalten in der Vereinigungsmenge

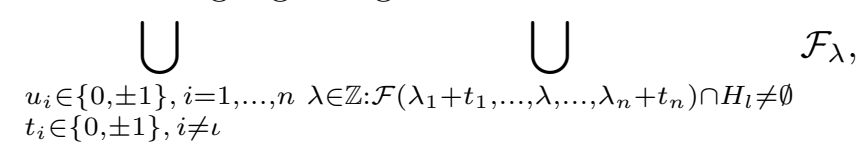

mit $\mathcal{F}_{\lambda}:=\mathcal{F}\left(\lambda_{1}+t_{1}+u_{1}, \ldots, \lambda+u_{\iota}, \ldots, \lambda_{n}+t_{n}+u_{n}\right)$, die wegen (58) aus höchstens $3^{n} \cdot 3^{n-1} \cdot n$ zu $\mathcal{F}$ kongruenten Parallelotopen besteht. Eine Volumenbetrachtung liefert dann $\left|M\left(x^{\prime}\right)\right| \leq 3^{n} \cdot 3^{n-1} \cdot n$. Mit (57), (59), (60) und (61) folgt

$$
R \ll\left(V^{(n-1) / n}+1\right) E .
$$

Standardüberlegungen ergeben

$$
\left|\left\{y \in \Gamma^{\prime} \mid y+\mathcal{F} \subset \dot{Q}\right\}\right|=\frac{\operatorname{vol}(Q)}{\operatorname{vol}(\mathcal{F})}+O\left(\left|\left\{y \in \Gamma^{\prime} \mid(y+\mathcal{F}) \cap \partial Q \neq \emptyset\right\}\right|\right) .
$$

$\widetilde{\mathcal{F}}:=\left\{\sum_{m=1}^{n} \mu_{m} x_{m} \mid \mu_{m} \in[0,1), m=1, \ldots, n\right\}$ ist Fundamentalparallelotop von $\Gamma . \mathcal{M}:=\mathcal{F} \cap \Gamma$ ist vollständiges Repräsentantensystem von $\Gamma / \Gamma^{\prime}$ und es ist $\mathcal{F}=\dot{\bigcup}_{x \in \mathcal{M}}(x+\widetilde{\mathcal{F}}) . \quad$ Mit $Q^{\prime}:=\prod_{l=1}^{n}\left(C_{l}+d, D_{l}-d\right)$, $Q^{\prime \prime}:=\prod_{l=1}^{n}\left[C_{l}-d, D_{l}+d\right]$ ( $d$ sei der Durchmesser von $\widetilde{\mathcal{F}}$ bezüglich der Standard-Maximumnorm) gilt

$$
\begin{aligned}
\mid\{y & \left.\in \Gamma^{\prime} \mid(y+\mathcal{F}) \cap \partial Q \neq \emptyset\right\} \mid \\
& \leq|\{z \in \Gamma \mid(z+\widetilde{\mathcal{F}}) \cap \partial Q \neq \emptyset\}| \leq\left|\left\{z \in \Gamma \mid z+\widetilde{\mathcal{F}} \subset Q^{\prime \prime} \backslash Q^{\prime}\right\}\right| \\
& \leq \frac{\operatorname{vol}\left(Q^{\prime \prime} \backslash Q^{\prime}\right)}{\operatorname{vol}(\widetilde{\mathcal{F}})} \ll\left(\max _{1 \leq l \leq n}\left(D_{l}-C_{l}\right)+1\right)^{n-1} \ll V^{(n-1) / n}+1 .
\end{aligned}
$$

Es ist $\operatorname{vol}(\mathcal{F})=|\mathcal{M}| \operatorname{vol}(\widetilde{\mathcal{F}}),|\mathcal{M}|=\left|a_{11} \ldots a_{n n}\right|=N(4 \mathfrak{a})$,

$$
\operatorname{vol}(\widetilde{\mathcal{F}})^{2}=\operatorname{det}\left(\sigma_{i}\left(\alpha_{j}\right)\right)^{2}=\left|d_{k / \mathbb{Q}}\right| \quad \text { und } \quad \sum_{x \in \mathcal{M}} \Phi(x)=\sum_{a \in \mathcal{M}(\mathfrak{a})}\left(\frac{f_{v, i, j}(a)}{\mathfrak{a}}\right) .
$$

Aus Lemma 4.3 folgt

$$
\sum_{a \in \mathcal{M}(\mathfrak{a})}\left(\frac{f_{v, i, j}(a)}{\mathfrak{a}}\right) \ll N(\mathfrak{a}) N(K(\mathfrak{a}))^{-1 / 2} 2^{\omega(\mathfrak{a})} \ll E
$$

und mit der $\Gamma^{\prime}$-Periodizität von $\Phi(x)$ und (56)

$$
\begin{aligned}
T= & \sum_{y \in \Gamma^{\prime}: y+\mathcal{F} \subset \dot{Q}}\left(\sum_{x-y \in \Gamma \cap \mathcal{F}} \Phi(x-y)\right)+O\left(\left(V^{(n-1) / n}+1\right) E\right) \\
= & \left(\sum_{a \in \mathcal{M}(\mathfrak{a})}\left(\frac{f_{v, i, j}(a)}{\mathfrak{a}}\right)\right)\left(\frac{V}{N(4 \mathfrak{a})\left|d_{k / \mathbb{Q}}\right|^{1 / 2}}+O\left(V^{(n-1) / n}+1\right)\right) \\
& +O\left(\left(V^{(n-1) / n}+1\right) E\right)
\end{aligned}
$$

und damit die Behauptung. 
Man definiere

$$
\begin{gathered}
c(v, \mathfrak{a}):=\sum_{j=1}^{L} c_{j}(v, \mathfrak{a}), \quad s(v):=\sum_{j=1}^{L} s_{j}(v), \\
c:=16^{-n}\left|d_{k / \mathbb{Q}}\right|^{-1 / 2}(m+1)^{-1}\left(\int_{-2}^{2}\left(4-\tau^{2}\right)^{m / 2} d \tau\right)^{n-1} .
\end{gathered}
$$

Lemma 4.8. Für $0 \neq v \in \mathcal{O}_{k}, \mathfrak{a} \neq 0$ ganzes Ideal und $x>2$ gilt

$$
\begin{array}{cc}
\sum_{D \in \mathcal{O}_{k} \text { Diskr., } u \in \mathcal{O}_{k}: u^{2}-D v^{2}=4} & \left|N_{k / \mathbb{Q}}(D)\right|^{m / 2} \chi_{D}(\mathfrak{a}) \\
=\frac{c \cdot c(v, \mathfrak{a}) x^{m+1}}{N(\mathfrak{a}) N(v)^{m+2}}+O\left(\frac{s(v)}{N(v)^{m}} \frac{N(\mathfrak{a})^{1+\varepsilon}}{N(K(\mathfrak{a}))^{1 / 2}} x^{m+(n-1) / n}\right)
\end{array}
$$

und

$$
c(v, \mathfrak{a}) \ll s(v) \frac{N(\mathfrak{a})^{1+\varepsilon}}{N(K(\mathfrak{a}))^{1 / 2}} .
$$

B ew eis. Die zweite Behauptung folgt mit Lemma 4.7 unmittelbar aus den Definitionen.

Für $A_{l}<B_{l}, l=1, \ldots, n, V:=\prod_{l=1}^{n}\left(B_{l}-A_{l}\right), 1 \leq j \leq L$, folgt aus (46) und Lemma 4.7

$$
\begin{aligned}
& \sum_{\substack{u, D \in \mathcal{O}_{k}: \\
D \equiv D_{j}(4), u^{2}-D v^{2}=4 \\
A_{1}<\sigma_{1}(u) \leq B_{1}, A_{l}<\sigma_{l}(u)<B_{l}, l=2, \ldots, n}} \chi_{D}(\mathfrak{a}) \\
& =\sum_{i=1}^{s_{j}(v)} \sum_{\substack{A_{1}<\sigma_{1}\left(m_{i j}+4 v^{2} t\right) \leq B_{1} \\
A_{l}<\sigma_{l}\left(m_{i j}+4 v^{2} t\right)<B_{l}, l=2, \ldots, n}}\left(\frac{\left(\left(m_{i j}+4 v^{2} t\right)^{2}-4\right) / v^{2}}{\mathfrak{a}}\right) \\
& =\sum_{i=1}^{s_{j}(v)} \sum_{\substack{t \in \mathcal{O}_{k}: \\
\frac{A_{1}-\sigma_{1}\left(m_{i j}\right)}{\sigma_{1}\left(4 v^{2}\right)}<\sigma_{1}(t) \leq \frac{B_{1}-\sigma_{1}\left(m_{i j}\right)}{\sigma_{1}\left(4 v^{2}\right)}}}^{\frac{A_{l}-\sigma_{l}\left(m_{i j}\right)}{\sigma_{l}\left(v^{2}\right)}<\sigma_{l}(t)<\frac{B_{l}-\sigma_{l}\left(m_{i j}\right)}{\sigma_{l}\left(4 v^{2}\right)}, l=2, \ldots, n} \\
& =\frac{c_{j}(v, \mathfrak{a}) V}{N\left(4 v^{2}\right) N(4 \mathfrak{a})\left|d_{k / \mathbb{Q}}\right|^{1 / 2}}+O\left(\left(V^{(n-1) / n}+1\right) s_{j}(v) \frac{N(\mathfrak{a})^{1+\varepsilon}}{N(K(\mathfrak{a}))^{1 / 2}}\right) .
\end{aligned}
$$

Durch $n$-malige Anwendung der partiellen Summation wird daraus

$$
\sum_{\substack{D \equiv D_{j}(4), u^{2}-D v^{2}=4 \\ u, D \in \mathcal{O}_{k}: \\ 2<\sigma_{1}(u) \leq x,-2<\sigma_{l}(u)<2, l=2, \ldots, n}} \chi_{D}(\mathfrak{a})\left|\sigma_{1}(u)^{2}-4\right|^{m / 2} \ldots\left|\sigma_{n}(u)^{2}-4\right|^{m / 2}
$$




$$
\begin{array}{r}
=\int_{2}^{x}\left|\tau^{2}-4\right|^{m / 2} d \tau\left(\int_{-2}^{2}\left|\tau^{2}-4\right|^{m / 2} d \tau\right)^{n-1} \frac{c_{j}(v, \mathfrak{a})}{N\left(4 v^{2}\right) N(4 \mathfrak{a})\left|d_{k / \mathbb{Q}}\right|^{1 / 2}} \\
+O\left(x^{m+(n-1) / n} s_{j}(v) \frac{N(\mathfrak{a})^{1+\varepsilon}}{N(K(\mathfrak{a}))^{1 / 2}}\right) .
\end{array}
$$

Einsetzen von $\varrho^{m / 2}=(\varrho+4)^{m / 2}+O\left(\varrho^{m / 2-1}\right), \varrho \geq 1$ und $N(D)=N\left(v^{2}\right)^{-1} \times$ $\prod_{l=1}^{n}\left|\sigma_{l}(u)^{2}-4\right|$ und Aufsummieren über $j$ liefert die erste Behauptung.

Sei $D \in \mathcal{O}_{k}$ Diskriminante, kein Quadrat. Nach Landau [10] gilt die verallgemeinerte Pólya-Vinogradov-Abschätzung

$$
\sum_{N(\mathfrak{a}) \leq x} \chi_{D}(\mathfrak{a}) \ll N(D)^{1 /(n+1)} \log ^{n} N(D) x^{(n-1) /(n+1)}, \quad x \geq 1 .
$$

Mit einer geeigneten Konstante $c_{4}(n)>0$ folgert man daraus wie bei Dirichletschen $L$-Reihen

$$
\begin{aligned}
& L\left(s, \chi_{D}\right) \ll(|t|+1) \log (N(D)+2) \\
& \text { für } s=\sigma+i t, 1-c_{4} / \log (N(D)+2) \leq \sigma \leq 1 .
\end{aligned}
$$

Lemma 2.6 von Hinz [7] lautet

$$
\begin{aligned}
& L\left(s, \chi_{D}\right) \ll N(D)^{1 / 2}(|t|+1)^{n / 2} \log (N(D)(|t|+2)), \\
& \quad 0 \leq \sigma \leq 2, t \in \mathbb{R} .
\end{aligned}
$$

Die folgenden Überlegungen verlaufen wie bei Barban [1], $\S 5$.

Seien $1 / 2<\gamma<1, \varepsilon>0$. Die $O$-Konstanten dürfen von $\gamma$ und $\varepsilon$ abhängen. Seien $N, x \geq 1$. Aus der Stirlingschen Formel folgt

$$
\Gamma(s-1)=\frac{\Gamma(s)}{s-1} \ll \frac{e^{-c_{5}|t|}}{|s-1|}, \quad 1 / 2 \leq \sigma \leq 2, t \in \mathbb{R}, s \neq 1 .
$$

Mit dem Residuensatz folgt

$$
\begin{aligned}
& \frac{1}{2 \pi i} \int_{2-i \infty}^{2+i \infty} \Gamma(s-1) L^{m}\left(s, \chi_{D}\right) N^{s-1} d s \\
&=L^{m}\left(1, \chi_{D}\right)+\frac{1}{2 \pi i} \int_{\gamma-i \infty}^{\gamma+i \infty} \Gamma(s-1) L^{m}\left(s, \chi_{D}\right) N^{s-1} d s .
\end{aligned}
$$

Die Dirichlet-Reihe

$$
L^{m}\left(s, \chi_{D}\right)=\sum_{\mathfrak{a}} \frac{\chi_{D}(\mathfrak{a}) \tau_{m}(\mathfrak{a})}{N(\mathfrak{a})^{s}}
$$

konvergiert absolut in der Halbebene $\Re s>1$ und gleichmäßig auf $\Re s=2$. Mit der Mellinschen Formel 


$$
\frac{1}{2 \pi i} \int_{a-i \infty}^{a+i \infty} \Gamma(s) u^{-s} d s=e^{-u} \quad(a, u>0)
$$

und

$$
I(D):=\frac{1}{2 \pi i} \int_{\gamma-i \infty}^{\gamma+i \infty} \Gamma(s-1) L^{m}\left(s, \chi_{D}\right) N^{s-1} d s
$$

folgt

$$
L^{m}\left(1, \chi_{D}\right)=\sum_{\mathfrak{a}} \frac{\chi_{D}(\mathfrak{a}) \tau_{m}(\mathfrak{a})}{N(\mathfrak{a})} e^{-N(\mathfrak{a}) / N}-I(D) .
$$

$I(D)$ ist von $\gamma$ unabhängig, da es die beiden anderen Terme sind. Wählt man für den Augenblick $\gamma:=1-c_{4} / \log (N(D)+2)$, so folgt aus (63)

$$
I(D) \ll \log ^{m+1}(N(D)+2) .
$$

(33), Proposition 2.4 und (66) liefern mit $\widetilde{c}:=\left((1 / \pi)^{n-1}\left|d_{k / \mathbb{Q}}\right|^{1 / 2}\right)^{m}$

(68) $\quad S_{m}(x)$

$$
\begin{aligned}
= & \widetilde{c} \sum_{D \in \mathcal{O}_{k} \text { Diskr., } u \in \mathcal{O}_{k}, v \in \mathcal{V}:}\left(N(D)^{1 / 2} L\left(1, \chi_{D}\right)\right)^{m} \\
= & \widetilde{c} \sum_{\mathfrak{a}} \frac{\tau_{m}(\mathfrak{a})}{N(\mathfrak{a})} e^{-N(\mathfrak{a}) / N} \sum_{v \in \mathcal{V}: N(v) \leq \sigma^{2}=4, N(v) \leq 2^{n-1} x} \sum_{D, u} N(D)^{m / 2} \chi_{D}(\mathfrak{a}) \\
& -\widetilde{c} \sum_{v \in \mathcal{V}: N(v) \leq 2^{n-1} x} \sum_{D, u} N(D)^{m / 2} I(D) \\
= & H(x)-R(x)
\end{aligned}
$$

wobei in den inneren Summen über alle Diskriminanten $D \in \mathcal{O}_{k}$ und alle $u \in \mathcal{O}_{k}$ summiert wird mit $u^{2}-D v^{2}=4,2<\sigma_{1}(u) \leq x,\left|\sigma_{l}(u)\right|<2$, $l=2, \ldots, n$.

Lemma 4.9. Mit $C:=c \widetilde{c}$ gilt

$$
\begin{aligned}
H(x)= & C x^{m+1} \sum_{v \in \mathcal{V}, \mathfrak{a} \neq 0} \frac{\tau_{m}(\mathfrak{a}) c(v, \mathfrak{a})}{N(\mathfrak{a})^{2} N(v)^{m+2}} \\
& +O\left(x^{m+1} N^{-1 / 2+\varepsilon}+N^{1 / 2+\varepsilon} x^{m+(n-1) / n+\varepsilon}\right) .
\end{aligned}
$$

B ew e is. Die beiden inneren Summen in $H(x)$ lassen sich mit Lemma 4.8 asymptotisch berechnen zu

$$
\frac{c x^{m+1}}{N(\mathfrak{a})} \sum_{v \in \mathcal{V}: N(v) \leq 2^{n-1} x} \frac{c(v, \mathfrak{a})}{N(v)^{m+2}}
$$




$$
+O\left(\frac{N(\mathfrak{a})^{1+\varepsilon}}{N(K(\mathfrak{a}))^{1 / 2}} x^{m+(n-1) / n} \sum_{v \in \mathcal{V}: N(v) \leq 2^{n-1} x} \frac{s(v)}{N(v)^{m}}\right) .
$$

Lemma 4.8 liefert mit (45)

$$
c(v, \mathfrak{a}) \ll N(v)^{\varepsilon} N(\mathfrak{a})^{1+\varepsilon} N(K(\mathfrak{a}))^{-1 / 2} .
$$

Mit

$$
\sum_{v \in \mathcal{V}: y<N(v) \leq z} 1 \leq \sum_{\mathfrak{b} \text { ganzes Ideal: } y<N(\mathfrak{b}) \leq z} 1 \ll z
$$

und partieller Summation ergibt sich daraus

$$
\begin{gathered}
\sum_{v \in \mathcal{V}: N(v)>y} \frac{c(v, \mathfrak{a})}{N(v)^{m+2}} \ll \frac{N(\mathfrak{a})^{1+\varepsilon}}{N(K(\mathfrak{a}))^{1 / 2}} y^{-m-1+\varepsilon}, \\
\sum_{v \in \mathcal{V}: N(v) \leq 2^{n-1} x} \frac{s(v)}{N(v)^{m}} \ll x^{\varepsilon} .
\end{gathered}
$$

Damit folgt

$$
\begin{aligned}
(70) H(x)= & \widetilde{c} \sum_{\mathfrak{a}} \frac{\tau_{m}(\mathfrak{a})}{N(\mathfrak{a})} e^{-N(\mathfrak{a}) / N}\left(\frac { c x ^ { m + 1 } } { N ( \mathfrak { a } ) } \left(\sum_{v \in \mathcal{V}} \frac{c(v, \mathfrak{a})}{N(v)^{m+2}}\right.\right. \\
& \left.\left.+O\left(\frac{N(\mathfrak{a})^{1+\varepsilon}}{N(K(\mathfrak{a}))^{1 / 2}} x^{-m-1+\varepsilon}\right)\right)+\frac{N(\mathfrak{a})^{1+\varepsilon}}{N(K(\mathfrak{a}))^{1 / 2}} x^{m+(n-1) / n+\varepsilon}\right) \\
= & C x^{m+1} \sum_{\mathfrak{a}} \frac{\tau_{m}(\mathfrak{a})}{N(\mathfrak{a})^{2}} e^{-N(\mathfrak{a}) / N} \sum_{v \in \mathcal{V}} \frac{c(v, \mathfrak{a})}{N(v)^{m+2}} \\
& +O\left(\sum_{\mathfrak{a}} \frac{N(\mathfrak{a})^{2 \varepsilon}}{N(K(\mathfrak{a}))^{1 / 2}} e^{-N(\mathfrak{a}) / N} x^{m+(n-1) / n+\varepsilon}\right) .
\end{aligned}
$$

Seien $\mathfrak{p}_{1}, \ldots, \mathfrak{p}_{t}$ paarweise verschiedene Primideale in $\mathcal{O}_{k}, \mathfrak{c}:=\mathfrak{p}_{1} \ldots \mathfrak{p}_{t}$. Für $y \geq 1$ gilt dann

$$
\begin{aligned}
|\{\mathfrak{a} \mid N(\mathfrak{a}) \leq y, K(\mathfrak{a})=\mathfrak{c}\}| & =\mid\left\{\mathfrak{c} \mathfrak{b}^{2} \mid N(\mathfrak{c} \mathfrak{b})^{2} \leq y, \mathfrak{b} \text { ganz }\right\} \mid \\
& =\left|\left\{\mathfrak{b} \mid N(\mathfrak{b}) \leq(y / N(\mathfrak{c}))^{1 / 2}\right\}\right| \ll(y / N(\mathfrak{c}))^{1 / 2}, \\
\sum_{N(\mathfrak{a}) \leq y} \frac{1}{N(K(\mathfrak{a}))^{1 / 2}} & =\sum_{\mathfrak{c} \text { quadratfrei: } N(\mathfrak{c}) \leq y} N(\mathfrak{c})^{-1 / 2} \sum_{\mathfrak{a}: N(\mathfrak{a}) \leq y, K(\mathfrak{a})=\mathfrak{c}} 1 \\
& \ll \sum_{\mathfrak{c}: N(\mathfrak{c}) \leq y} \frac{1}{N(\mathfrak{c})^{1 / 2}}\left(\frac{y}{N(\mathfrak{c})}\right)^{1 / 2} \ll y^{1 / 2} \log (y+2) .
\end{aligned}
$$

Daraus folgt durch partielle Summation

$$
\sum_{\mathfrak{a}} \frac{N(\mathfrak{a})^{2 \varepsilon}}{N(K(\mathfrak{a}))^{1 / 2}} e^{-N(\mathfrak{a}) / N} \ll N^{1 / 2+3 \varepsilon},
$$




$$
\begin{aligned}
& \sum_{c \in \mathcal{V}, \mathfrak{a}} \frac{\tau_{m}(\mathfrak{a}) c(v, \mathfrak{a})}{N(\mathfrak{a})^{2} N(v)^{m+2}}\left(e^{-N(\mathfrak{a}) / N}-1\right) \\
& \ll \sum_{v \in \mathcal{V}, \mathfrak{a}} \frac{\min \{N(\mathfrak{a}) / N, 1\}}{N(K(\mathfrak{a}))^{1 / 2} N(\mathfrak{a})^{1-2 \varepsilon} N(v)^{m+2-\varepsilon}} \\
&=\sum_{v \in \mathcal{V}} \frac{1}{N(v)^{m+2-\varepsilon}}\left(\sum_{N(\mathfrak{a}) \leq N}+\sum_{N(\mathfrak{a})>N}\right) \ll N^{-1 / 2+2 \varepsilon} .
\end{aligned}
$$

Einsetzen in (70) liefert die Behauptung.

Das folgende Lemma wird für Dirichlet-Reihen in Barban [1], Lemma 5.3, angegeben und in Titchmarsh [25], Theorem 14.2, für die Riemannsche Zetafunktion unter der Voraussetzung der Riemannschen Vermutung bewiesen. Mit den gleichen Hilfsmitteln und (64) erhält man die hier benötigte Version.

Lemma 4.10. Sei $D \in \mathcal{O}_{k}$ Diskriminante, kein Quadrat, $1 / 2<\sigma_{0}<$ $1, B \geq 1$. L $L\left(s, \chi_{D}\right)$ habe im Gebiet $\left\{\sigma+i t\left|\sigma \geq \sigma_{0},\right| t \mid \leq B\right\}$ keine Nullstellen. Dann gilt für $s=\sigma+i t, \sigma_{0}+\left(1-\sigma_{0}\right) /\left(c_{7} \log \log ((|t|+3) N(D))\right) \leq$ $\sigma \leq 1,|t|+c_{6} \log \log ((|t|+3) N(D)) \leq B-2$,

$$
\begin{aligned}
& \left|\log L\left(s, \chi_{D}\right)\right| \\
& \quad \leq \frac{c_{8}}{1-\sigma_{0}} \log \log ((|t|+3) N(D))\{\log ((|t|+3) N(D))\}^{(1-\sigma) /\left(1-\sigma_{0}\right)} .
\end{aligned}
$$

Für einen Idealklassencharakter $\chi \bmod \mathfrak{q}$ im engeren Sinn sei

$$
L(s, \chi):=\sum_{\mathfrak{a}} \frac{\chi(\mathfrak{a})}{N(\mathfrak{a})^{s}}, \quad \Re s>1,
$$

die zugehörige Heckesche $L$-Reihe. Für $\sigma>1 / 2, T \geq 2$, sei $N(\sigma, T, \chi)$ die Anzahl der Nullstellen $\varrho=\beta+i t$ von $L(s, \chi)$ im Gebiet $\beta \geq \sigma,|t| \leq T$ und $R(\sigma, T):=\{\beta+i t|1 \geq \beta \geq \sigma| t \mid, \leq T\}$. Aus Satz B von Hinz [7] folgt:

$\mid\{\chi \bmod \mathfrak{q} \mid \chi$ primitiv, $N(\mathfrak{q}) \leq Q, L(s, \chi)$ hat in $R(\sigma, T)$ eine Nullstelle $\} \mid$

$$
\ll T^{A(n)} \log ^{B(n)} Q \cdot Q^{4(1-\sigma) / \sigma}, \quad 3 / 4 \leq \sigma \leq 1, T \geq 2, Q \geq 1 .
$$

Lemma 4.11. Für $3 / 4 \leq \sigma \leq 1, T, x \geq 2, \varepsilon>0$ gilt

$$
\begin{aligned}
& A(x, \sigma, T) \\
& \quad \begin{array}{l}
:=\mid\{(D, u, v) \mid \\
D
\end{array} \\
& \quad 2<\sigma_{1}(u) \leq x,\left|\sigma_{l}(u)\right|<2, l=2, \ldots, n, \\
& \left.L\left(s, \chi_{D}\right) \text { hat in } R(\sigma, T) \text { eine Nullstelle }\right\} \mid \\
& \ll x^{8(1-\sigma) / \sigma+\varepsilon} T^{A(n)} .
\end{aligned}
$$


B ew eis. $(D, u, v)$ liege in der betrachteten Menge. Für $K:=k(\sqrt{D})$ existiert nach (8) ein ganzes Ideal $\mathfrak{c}$ mit $D \mathcal{O}_{k}=d_{K / k} \mathfrak{c}^{2}$, d.h.

$$
N\left(d_{K / k}\right) \leq N(D)=N(v)^{-2} \prod_{l=1}^{n}\left|\sigma_{l}\left(u^{2}-4\right)\right| \leq 4^{n-1} x^{2} .
$$

Nach Lemma 2.1 ist

$$
L\left(s, \chi_{D}\right)=L\left(s, \chi_{D}^{*}\right) \prod_{\mathfrak{p} \mid D}\left(1-\frac{\chi_{D}^{*}(\mathfrak{p})}{N(\mathfrak{p})^{s}}\right),
$$

d.h. auch $L\left(s, \chi_{D}^{*}\right)$ hat eine Nullstelle in $R(\sigma, T)$. Aus (37), (35), (39), (41), Lemma 2.3 und (4) folgt

$$
\begin{aligned}
& A(x, \sigma, T) \leq \quad \sum \quad\left|S_{K}\right| \\
& K: \begin{array}{l}
\text { Es gibt } D_{1} \in \widetilde{D} \text { mit } K=k\left(\sqrt{D_{1}}\right), N\left(d_{K / k}\right) \leq 4^{n-1} x^{2}, \\
L\left(s, \chi_{D_{1}}^{*}\right) \text { hat in } R(\sigma, T) \text { eine Nullstelle }
\end{array}
\end{aligned}
$$

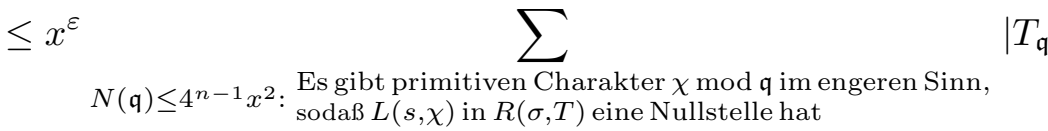

$$
\begin{aligned}
& \ll x^{\varepsilon} T^{A(n)} \log ^{B(n)} x^{2} \cdot\left(x^{2}\right)^{4(1-\sigma) / \sigma} .
\end{aligned}
$$

Lemma 4.12. Sei $3 / 4<\sigma_{0}<\gamma<1, \varepsilon>0, x \geq x_{0}\left(\sigma_{0}, \gamma, \varepsilon\right), N \geq 1$. Dann gilt

$$
R(x) \ll_{\sigma_{0}, \gamma, \varepsilon} x^{m+\varepsilon}\left(x^{8\left(1-\sigma_{0}\right) / \sigma_{0}}+x N^{\gamma-1}\right) .
$$

B ew eis. Für $D_{0} \in \mathcal{D}, D_{0} \mathcal{O}_{k}=\mathfrak{a}$ ist die Abbildung

$$
\phi:\left\{D \in \mathcal{D} \mid D \mathcal{O}_{k}=\mathfrak{a}\right\} \rightarrow E\left(\mathcal{O}_{k}\right) / E\left(\mathcal{O}_{k}\right)^{2}, \quad D \rightarrow D D_{0}^{-1} \bmod E\left(\mathcal{O}_{k}\right)^{2},
$$

injektiv und damit

$$
\left|\left\{D \in \mathcal{D} \mid D \mathcal{O}_{k}=\mathfrak{a}\right\}\right| \ll 1 .
$$

Sei $D \in \mathcal{O}_{k}$ Diskriminante, kein Quadrat, $c_{9}\left(\sigma_{0}, \gamma\right) \leq N(D) \leq c_{10} x^{2}$, und $L\left(s, \chi_{D}\right)$ habe in $R\left(\sigma_{0}, \log ^{2} x\right)$ keine Nullstelle. Nach Lemma 4.10 ist dann

$$
L\left(s, \chi_{D}\right) \ll x^{\varepsilon} \quad \text { für } s=\gamma+i t,|t| \leq \frac{1}{2} \log ^{2} x, x \geq x_{0}\left(\sigma_{0}, \gamma, \varepsilon\right) .
$$

Nach (64) ist

$$
L\left(s, \chi_{D}\right) \ll(|t|+2)^{n / 2+\varepsilon} x^{1+\varepsilon} \quad \text { für } s=\gamma+i t,|t| \geq \frac{1}{2} \log ^{2} x .
$$

Mit (65) folgt daraus

$$
\begin{aligned}
I(D) & \ll \int_{-\infty}^{\infty}|\Gamma(\gamma-1+i t)|\left|L\left(\gamma+i t, \chi_{D}\right)\right|^{m} N^{\gamma-1} d t \\
& =\int_{|t| \leq\left(\log ^{2} x\right) / 2}+\int_{|t| \geq\left(\log ^{2} x\right) / 2} \ll x^{m \varepsilon} N^{\gamma-1} .
\end{aligned}
$$


Aus (68) folgt

$$
R(x) \ll x^{m}\left(\sum_{1}|I(D)|+\sum_{2}|I(D)|+\sum_{3}|I(D)|\right) .
$$

Dabei wird in der dritten Summe summiert über $D \in \mathcal{O}_{k}$ Diskriminante, $u \in \mathcal{O}_{k}, v \in \mathcal{V}$, mit $u^{2}-D v^{2}=4,2<\sigma_{1}(u) \leq x,\left|\sigma_{l}(u)\right|<2, l=2, \ldots, n$, und der zusätzlichen Bedingung:

$$
N(D)>c_{9}\left(\sigma_{0}, \gamma\right) \quad \text { und } \quad L\left(s, \chi_{D}\right) \text { hat in } R\left(\sigma_{0}, \log ^{2} x\right) \text { eine Nullstelle. }
$$

Mit (67) und Lemma 4.11 ist also

$$
\sum_{3}|I(D)| \ll \log ^{m+1} x A\left(x, \sigma_{0}, \log ^{2} x\right) \ll x^{8\left(1-\sigma_{0}\right) / \sigma_{0}+\varepsilon} .
$$

In der ersten Summe lautet die zusätzliche Bedingung: $N(D) \leq c_{9}\left(\sigma_{0}, \gamma\right)$, und in der zweiten Summe: $N(D)>c_{9}\left(\sigma_{0}, \gamma\right), L\left(s, \chi_{D}\right)$ hat in $R\left(\sigma_{0}, \log ^{2} x\right)$ keine Nullstelle. Mit (67), (35), (34), (40) und (71) ist

$$
\begin{aligned}
\sum_{1}|I(D)| & \sum_{D \in \widetilde{\mathcal{D}}, u \in \mathcal{O}_{k}, v \in \mathcal{V}: u^{2}-D v^{2}=4,2<\sigma_{1}(u) \leq x, N(D) \ll 1} \log ^{m+1}(N(D)+2) \\
& \ll \sum_{D \in \mathcal{D}, u \in \mathcal{O}_{k}, v \in \mathcal{V}, e \in E\left(\mathcal{O}_{k}\right): u^{2}-D(e v)^{2}=4,2<\sigma_{1}(u) \leq x, N(D) \ll 1} 1 \\
& =\sum_{D \in \mathcal{D}, u, v \in \mathcal{O}_{k}: u^{2}-D v^{2}=4,2<\sigma_{1}(u) \leq x, N(D) \ll 1} 1 \\
& =\sum_{D \in \mathcal{D}: N(D) \ll 1} \sum_{\alpha \in U_{D}: 1<\alpha \leq x} 2 \ll \sum_{D \in \mathcal{D}: N(D) \ll 1} \frac{\log x}{\log \varepsilon_{D}} \ll \log x .
\end{aligned}
$$

Auf die gleiche Weise ergibt sich mit (72)

$$
\sum_{2}|I(D)| \ll x^{m \varepsilon} N^{\gamma-1} \sum_{\substack{2<\sigma_{1}(u) \leq x \\ u \in \mathcal{O}_{k}: \\\left|\sigma_{l}(u)\right|<2, l=2, \ldots, n}} \sum_{D \in \mathcal{D}, v \in \mathcal{O}_{k}: u^{2}-D v^{2}=4} 1 .
$$

Mit der Definition von $\mathcal{D}$ ist die innere Summe

$$
\leq \sum_{\mathfrak{d}, \mathfrak{v}: \mathfrak{d} \mathfrak{v}^{2}=\left(u^{2}-4\right) \mathcal{O}_{k}} 2 \leq 2 \tau_{2}\left(\left(u^{2}-4\right) \mathcal{O}_{k}\right) \ll x^{\varepsilon},
$$

d.h.

$$
\sum_{2}|I(D)| \ll x^{m \varepsilon} N^{\gamma-1} x^{\varepsilon} \sum_{\substack{u \in \mathcal{O}_{k}: \\ 2<\sigma_{1}(u) \leq x \\\left|\sigma_{l}(u)\right|<2, l=2, \ldots, n}} 1 \ll x^{1+(m+1) \varepsilon} N^{\gamma-1},
$$

womit alles gezeigt ist. 
Beweis von Satz 1.1. Die optimale Wahl der Parameter lautet

$$
\begin{gathered}
N=x^{\alpha}, \quad \gamma=\sigma_{0}+\varepsilon, \quad \alpha=\frac{1}{n} \cdot \frac{1}{3 / 2-\sigma_{0}}, \\
\sigma_{0}=\frac{21.5-1 / n}{18-2 / n}-\sqrt{\left(\frac{21.5-1 / n}{18-2 / n}\right)^{2}-\frac{12}{9-1 / n} .}
\end{gathered}
$$

Mit $\varrho(n)=8\left(1-\sigma_{0}\right) / \sigma_{0}$ folgt dann aus (68), Lemma 4.9 und Lemma 4.12

$$
\begin{gathered}
S_{m}(x)=\lambda_{m} x^{m+1}+O_{\varepsilon}\left(x^{m+\varrho(n)+\varepsilon}\right), \quad x \geq x_{1}(n, \varepsilon), \\
\lambda_{m}:=C \sum_{v \in \mathcal{V}, \mathfrak{a}} \frac{\tau_{m}(\mathfrak{a}) c(v, \mathfrak{a})}{N(\mathfrak{a})^{2} N(v)^{m+2}} .
\end{gathered}
$$

Aus (44) und (43) ergibt sich damit $\Theta_{m}(x) \leq \widetilde{S}_{m}(x)=\lambda_{m} x^{m+1}+O\left(x^{m+\varrho+\varepsilon}\right)$ $\left(\ll x^{m+1}\right)$, und aus (40) folgt $\Theta_{m}(x)=0$ für $1 \leq x<\mu(k)$. (43) liefert schließlich

$$
\begin{aligned}
\Theta_{m}(x) & =\widetilde{S}_{m}(x)-\sum_{2 \leq l \leq \log x / \log \mu} \Theta_{m}\left(x^{1 / l}\right) \\
& =\lambda_{m} x^{m+1}+O\left(x^{m+\varrho+\varepsilon}\right)+O\left(\sum_{2 \leq l \leq \log x / \log \mu}\left(x^{1 / l}\right)^{m+1}\right) \\
& =\lambda_{m} x^{m+1}+O\left(x^{m+\varrho+\varepsilon}\right) .
\end{aligned}
$$

Bemerkung. Ist man mit einem etwas schlechteren $\varrho(n)$ zufrieden, so kann der größte Teil von Abschnitt 4 eingespart werden. Die Summe in Lemma 4.2 wird trivial durch $N(\mathfrak{p})$ abgeschätzt. Lemma 4.7 läßt sich dann viel einfacher mit dem Fehlerterm $O\left(\left(V^{(n-1) / n}+1\right) N(\mathfrak{a})\right)$ beweisen. Die zweite Abschätzung in Lemma 4.7 muß allerdings weiterhin benutzt werden, um eine nichttriviale Abschätzung für $c(v, \mathfrak{a})$ zu erhalten, die dann in Lemma 4.9 verwendet wird. In diesem Fall lauten die optimalen Parameter

$$
\begin{gathered}
N=x^{\alpha}, \quad \gamma=\sigma_{0}+\varepsilon, \quad \alpha=\frac{1}{n} \cdot \frac{1}{2-\sigma_{0}}, \\
\sigma_{0}=\frac{26-1 / n}{18-2 / n}-\sqrt{\left(\frac{26-1 / n}{18-2 / n}\right)^{2}-\frac{16}{9-1 / n}} .
\end{gathered}
$$

Der Wert für $\varrho(n)=8\left(1-\sigma_{0}\right) / \sigma_{0}$ ist stets größer als der in (74) angegebene Wert.

Für $n=1$ z.B. ist $\varrho(1)=0.840$ mit und $\varrho(1)=0.908$ ohne Weilsche Charaktersummenabschätzungen.

Die Konstante $\lambda_{m}$. Die Berechnung des exakten Werts von $\lambda_{m}$ ist mühsam. Mit einer Rechnung wie bei Sarnak [18] (Berechnung von $b_{1}$ ) ergibt sich für $k=\mathbb{Q}, m=1: \lambda_{1}=1 / 2$.

Für $k=\mathbb{Q}, m>1$, kann $\lambda_{m}$ als unendliches Produkt dargestellt werden; einen einfachen Ausdruck konnte ich nicht finden. 
Für $k \neq \mathbb{Q}$ wird schon im Fall $m=1$ die Rechnung unübersichtlich, da die Lösungsanzahl von quadratischen Kongruenzen $\bmod \mathfrak{p}^{a}$ bestimmt werden muß; für $\mathfrak{p} \mid 2$ sind dafür Fallunterscheidungen nötig.

Im allgemeinen Fall kann $\lambda_{m}>0$ wie folgt gezeigt werden:

Wegen $S_{m}(x) \geq 0$ folgt $\lambda_{m} \geq 0$ aus (75). Wäre $\lambda_{m}=0$, so wäre einerseits

$$
S_{m}(x) \ll x^{m+\varrho+\varepsilon} .
$$

Andererseits gilt für eine Diskriminante $D \in \mathcal{O}_{k}$, kein Quadrat, $K:=$ $k(\sqrt{D})$,

$$
[K: \mathbb{Q}]=2 n, \quad T\left(E\left(\mathcal{O}_{K}\right)\right) \subset\left\{\xi \in \mathbb{C} \mid \xi^{m}=1, \phi(m) \leq 2 n\right\},
$$

d.h. $\left|T\left(E\left(\mathcal{O}_{K}\right)\right)\right| \ll 1$. Nach Lemma 2.12, (31), (28) und (29) ist

$$
\begin{aligned}
h(D) & =\frac{h_{K}}{h_{k}} \gamma(D) \frac{\left[E\left(\mathcal{O}_{k}\right): \operatorname{Bild} N_{K / k}\right]}{\left[\operatorname{Kern} N_{K / k}: U_{D}\right]}, \\
R(D) & =\frac{R_{K}}{R_{k}} \frac{\left|T\left(U_{D}\right)\right| \cdot\left[\operatorname{Kern} N_{K / k}: U_{D}\right] \cdot\left|T\left(E\left(\mathcal{O}_{k}\right)\right)\right|}{\left|T\left(E\left(\mathcal{O}_{K}\right)\right)\right| \cdot\left[E\left(\mathcal{O}_{k}\right): \operatorname{Bild} N_{K / k}\right]} .
\end{aligned}
$$

Mit Lemma 2.9 folgt daraus:

$$
h(D) R(D) \gg_{k} h_{K} R_{K} N(\mathfrak{c}) 2^{-\omega(\mathfrak{c})} \gg h_{K} R_{K} N(\mathfrak{c})^{1-\varepsilon} .
$$

Mit dem Satz von Brauer-Siegel (Stark [24], S. 968) und (8) ist damit

$$
\begin{aligned}
h(D) R(D) & \gg_{k, \varepsilon}\left|d_{K / \mathbb{Q}}\right|^{1 / 2-\varepsilon} N(\mathfrak{c})^{1-\varepsilon}=\left.\left.|| d_{k / \mathbb{Q}}\right|^{1 / 2} N_{k / \mathbb{Q}}\left(d_{K / k}\right)\right|^{1 / 2-\varepsilon} N(\mathfrak{c})^{1-\varepsilon} \\
& \gg_{k, \varepsilon} N(D)^{1 / 2-\varepsilon} .
\end{aligned}
$$

Wegen $c\left(1, \mathcal{O}_{k}\right)>0$ folgt aus Lemma 4.8 für $v=1, \mathfrak{a}=\mathcal{O}_{k}$

$$
\begin{aligned}
& S_{m}(x) \geq c_{18} \sum_{D \in \mathcal{O}_{k} \text { Diskr., } u \in \mathcal{O}_{k}:} \sum_{\substack{u^{2}-D=4 \\
2<\sigma_{1}(u) \leq x,\left|\sigma_{l}(u)\right|<2, l=2, \ldots, n}}\left(N(D)^{1 / 2-\varepsilon}\right)^{m} \\
& \geq c_{19}(\varepsilon) x^{m+1-2 \varepsilon m} \text {. }
\end{aligned}
$$

Ist $\varepsilon>0$ in Abhängigkeit von $\varrho$ und $m$ klein genug, so steht das zu (76) im Widerspruch.

\section{References}

[1] M. B. Barban, The "large sieve" method and its applications in the theory of numbers, Russian Math. Surveys 21 (1966), 49-103.

[2] S. I. Borewicz und I. R. Š afarevič, Zahlentheorie, Birkhäuser, 1966.

[3] G. L. Dirichlet, Recherches sur les formes quadratiques à coefficients et à indeterminés complexes, Premier partie, J. Reine Angew. Math. 24 (1842), 291-371.

[4] E. Hecke, Eine neue Art von Zetafunktionen und ihre Beziehungen zu Verteilung der Primzahlen, Math. Z. 1 (1918), 357-376; 6 (1920), 11-21.

[5] _ - Vorlesungen über die Theorie der algebraischen Zahlen, Akademische Verlagsgesellschaft, Leipzig, 1923; englische Übersetzung: Lectures on the Theory of Algebraic Numbers, Graduate Texts in Math. 77, Springer, 1981. 
[6] D. Hilbert, Über die Theorie des relativ-quadratischen Zahlkörpers, Math. Ann. 51 (1899), 1-127.

[7] J. Hinz, Über Nullstellen der Heckeschen Zetafunktionen in algebraischen Zahlkörpern, Acta Arith. 31 (1976), 167-193.

[8] C. Hooley, On the Pellian equation and the class number of indefinite binary quadratic forms, J. Reine Angew. Math. 353 (1984), 98-131.

[9] H. Iw aniec, Prime geodesic theorem, ibid. 349 (1984), 136-159.

[10] E. Landau, Verallgemeinerung eines Pólyaschen Satzes auf algebraische Zahlkörper, Nachr. Königl. Gesell. Wiss. Göttingen Math. Phys. Kl. 1918, 478-488.

[11] -, Über Ideale und Primideale in Idealklassen, Math. Z. 2 (1918), 52-154.

[12] S. Lang, Algebraic Number Theory, Addison-Wesley, 1970.

[13] F. Mertens, Über einige asymptotische Gesetze der Zahlentheorie, J. Reine Angew. Math. 77 (1874), 289-338.

[14] W. Narkiewicz, Elementary and Analytic Theory of Algebraic Numbers, Springer, 1990.

[15] O. T. O'Meara, Introduction to Quadratic Forms, Grundlehren Math. Wiss. 117, Springer, 1973.

[16] P. Sarnak, Class numbers of indefinite binary quadratic forms, J. Number Theory 15 (1982), 229-247.

[17] - , The arithmetic and geometry of some hyperbolic three manifolds, Acta Math. 151 (1983), 253-295.

[18] - Class numbers of indefinite binary quadratic forms II, J. Number Theory 21 (1985), 333-346.

[19] W. M. Schmidt, Equations over Finite Fields. An Elementary Approach, Lecture Notes in Math. 536, Springer, 1976.

[20] T. Shintani, On zeta-functions associated with the vector space of quadratic forms, J. Fac. Sci. Univ. Tokyo Sect. IA Math. 22 (1975), 25-65.

[21] J.-M. Shyr, Class numbers of binary quadratic forms over algebraic number fields, J. Reine Angew. Math. 307/308 (1979), 353-364.

[22] C. L. Siegel, The average measure of quadratic forms with given determinant and signature, Ann. of Math. 45 (1944), 667-685.

[23] A. Speiser, Die Theorie der binären quadratischen Formen mit Koeffizienten und Unbestimmten in einem beliebigen Zahlkörper, Dissertation, Göttingen, 1909.

[24] H. M. Stark, The analytic theory of algebraic numbers, Bull. Amer. Math. Soc. 81 (1975), 961-972.

[25] E. C. Titchmarsh, The Theory of the Riemann Zeta-Function, Clarendon Press, 1986.

[26] I. M. Vinogradov, A new method of deriving asymptotic expressions for arithmetic functions, Izv. Ross. Akad. Nauk (6) 16 (1917), 1347-1378.

[27] - On an asymptotic equality in the theory of quadratic forms, Zh. Fiz. Mat. Obshch. Perm. Univ. 1 (1918), 18-28.

[28] D. Wolke, Moments of class numbers III, J. Number Theory 4 (1972), 523-531.

[29] D. B. Zagier, Zetafunktionen und quadratische Körper, Springer Hochschultext, Springer, 1981.

FEDDERSTR. 5

79106 FREIBURG

DEUTSCHLAND 